\title{
A Voronoi Interface Approach to Cell Aggregate Electropermeabilization
}

\author{
Arthur Guittet $^{\mathrm{a}}$, C. Poignard ${ }^{\mathrm{c}}$, Frederic Gibou $^{\mathrm{a}, \mathrm{b}}$ \\ ${ }^{a}$ Department of Mechanical Engineering, University of California, Santa Barbara, CA 93106-5070 \\ ${ }^{b}$ Department of Computer Science, University of California, Santa Barbara, CA 93106-5110 \\ ${ }^{c}$ INRIA, F-33400 Talence, France. \\ Univ. Bordeaux, IMB, UMR 5251, F-33400 Talence, France \\ CNRS, IMB, UMR 5251, F-33400 Talence, France.
}

\begin{abstract}
We present a Voronoi Interface approach to the study of cell electropermeabilization. We consider the nonlinear electropermeabilization model of Poignard et al. [20], which takes into account the jump in the voltage potential across cells' membrane. The jump condition is imposed in a sharp manner, using the Voronoi Interface Method of Guittet et al. [14], while adaptive Quad-/Oc-tree grids are employed to automatically refine near the cells boundary for increased accuracy. Numerical results are provided to illustrate the accuracy of the methods. We also carry out simulations in three spatial dimensions to investigate the influence of shadowing and of the cells shape on the degree of permeabilization.
\end{abstract}

Keywords: Sharp methods, Finite Volume method, Cell modelling, Level-Set method, Quad/Oc-tree, Voronoi

2000 MSC: 65M08, 65M12, 92C37

\section{Introduction}

Electropermeabilization, also known as electroporation or electropulsation, is a significant increase in the permeability and in the electrical conductivity of the cell membrane that occurs when electric pulses of large amplitude (a few hundred volts per centimeter) are applied to cell membrane $[27,11,37]$. For high electric fields, the membrane is (reversibly or irreversibly) permeabilized, which enables the transfer of non-permeable molecules into the cell cytoplasm by diffusion through the electropermeabilized membrane areas. If the pulses are too long, too numerous, or if their amplitude is too high, the cell membrane is irreversibly destroyed and the cells are killed. Electroporation is important in the treatment of some cancers, as it provides an avenue to deliver therapeutic molecules directly into the cells of targeted areas.

Even though cell electropermeabilization is a well-known phenomenon, at least from the experimental point of view, there is a lack of predictive computational models that are validated

\footnotetext{
${ }^{*}$ Corresponding author: arthur.guittet@gmail.com
} 
by experiments $[34,33]$. This prevents a systematic use of electropermeabilization in configurations far from the experimental settings. This is particularly pertinent to deep-seated tumor treatments for which irreversible electropermeabilization (IRE) or electrochemotherapy (ECT) need an accurate distribution of the electroporated region. The lack of a predictive computational framework is mainly due to the complexity of the electropermeabilization models at the cell scale $[4,38,36,18,20]$, which are written in terms of partial differential equations in irregular geometries and that utilize non-standard transmission conditions through the cell's membrane. The reader may refer to the recent review [30] on different ways to model cell electroporation. In addition, the experimental settings are usually far from the standard numerical configurations since most of the simulations deal with one single cell, while in vitro experiments, a large number of cells (in suspension or aggregated in spheroids) is considered. Therefore, important phenomena, such as cell screening, or electropermeabilization at the mesoscale (scale of cell aggregates) cannot be accounted for by existing computational approaches.

The aim of this paper is to present an accurate numerical method that provides a first step in that direction by enabling the computation of the voltage potential in cell aggregates when an electropermeabilizing electric field is applied. A cell membrane is very thin and acts as a capacitor, thus leading to a discontinuity in the electric potential where the jump is proportional to the electric flux. A variety of methods exist to solve elliptic systems with discontinuities. The finite element method is one of the most popular approach [1, 2, 5, 17]. It is well studied and guarantees high accuracy and a symmetric positive definite linear system. However, its efficiency relies on the quality of the mesh that must body-fit the irregular domain's boundary. In contrast, interface capturing methods are based on Cartesian grids that are easily generated and impose the discontinuous boundary condition implicitly. The Immersed Interface Method [21] and its development, the Immersed Finite Element Method and the Immersed Finite Volume Method [22, $13,6]$, produce second-order accurate solutions in the $L^{\infty}$ norm but produce asymmetric linear system in the case of discontinuous diffusion coefficients. This is also the case for the interface treatment used in Mirzadeh et al. [28]. Cisterno and Weynans developed a second-order accurate method [3] and applied it to the electropermeabilization problem [19], though their method is not compact and leads to asymmetric linear systems. The philosophy of the Ghost Fluid Method [8], originally developed to treat in a sharp fashion shocks and contact discontinuities in compressible flows, has also been applied to discontinuous elliptic problems [23]. In this case, the resulting linear system is symmetric positive definite and the jump conditions only affect the right-hand side of the linear system. The method is first-order accurate (in the maximum norm) in the general case [24], although second-order accuracy (in the maximum norm) is achieved in the particular case of both a constant discontinuity in the solution and a constant diffusion coefficient. The drop to first-order accuracy comes from the dimension-by-dimension approach of the Ghost Fluid Method, leading to non-converging gradients in the general case. The Voronoi Interface Method, introduced in Guittet et al. [14], considers a capturing Voronoi mesh based on an underlying Cartesian grid before applying a one-dimensional ghost-fluid philosophy of [23]. As a result, the discretized fluxes are normal to the interface so that second-order accuracy is achieved in the general case. The gradients converge with first-order accuracy and the method preserves the symmetry positive definiteness of the linear system with only the right-hand side affected by the jump conditions. 
In this paper, we develop a VIM approach for the simulation of electropermeabilization for both single cells and spheroids. Multicellular tumor cells spheroids are particular cell aggregates that mimic the behavior of tumors [16], in particular from the electropermeabilization point of view [12]. Therefore, providing a computational framework that makes it possible to compute accurately the electric field in such cell aggregates is a crucial step forward for the clinical use of electropermeabilization-based therapies. In the first part of this article, we present the non-linear model for cell electropermeabilization before describing the numerical method. We then validate the computational method in two and three spatial dimensions and investigate the behavior of cell aggregates.

\section{Electrical model for a single cell}

Cell membranes are very thin and very resistive, therefore they are usually described as a two spatial dimensions surface, $\Gamma$, with given capacitance, $C$, and surface conductance, $S$, as described by Schwan, Stuchly et al. [10,7] and depicted in figure 1.

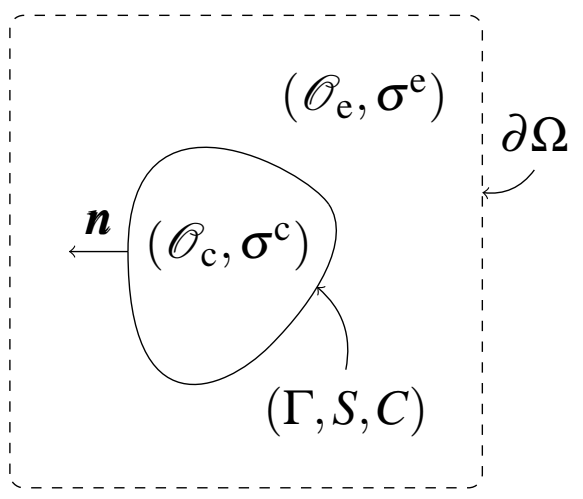

Figure 1: Geometry of the problem. The cell $\mathscr{O}_{\mathrm{c}}$ is imbedded in the extra-cellular matrix $\mathscr{O}_{\mathrm{e}}$. The entire domain $\Omega$ is defined by $\Omega=\mathscr{O}_{\mathrm{e}} \cup \overline{\mathscr{O}_{\mathrm{c}}}$.

Denoting by $\mathscr{O}_{\mathrm{c}}$ the cell cytoplasm and by $\mathscr{O}_{\mathrm{e}}$ the extracellular medium and defining by $\sigma$ the conductivity of the medium as:

$$
\sigma= \begin{cases}\sigma^{\mathrm{e}}, & \text { in } \mathscr{O}_{\mathrm{e}}, \\ \sigma^{\mathrm{c}}, & \text { in } \mathscr{O}_{\mathrm{c}},\end{cases}
$$

the electric potential satisfies the following boundary value problem:

$$
u(0, \cdot)=0 \quad \text { in } \mathscr{O}_{\mathrm{e}} \cup \mathscr{O}_{\mathrm{c}}
$$

and for any $t>0$,

$$
\begin{aligned}
& \Delta u=0, \quad \text { in }(0, T) \times\left(\mathscr{O}_{\mathrm{e}} \cup \mathscr{O}_{\mathrm{c}}\right), \\
& u(t, \cdot)=g(t, \cdot) \quad \text { on }(0,+\infty) \times \partial \Omega,
\end{aligned}
$$


with the jump conditions:

$$
\begin{aligned}
& {\left[\sigma \partial_{\mathbf{n}} u\right]=0, \quad \text { on }(0, T) \times \Gamma,} \\
& C \partial_{t}[u](t, \cdot)+S(t,[u])[u]=\left.\sigma \partial_{\mathbf{n}} u(t, \cdot)\right|_{\Gamma}, \quad \text { on }(0, T) \times \Gamma .
\end{aligned}
$$

The jump condition (1d) enforces the continuity of the flux and the transmission condition on the jump of the potential (1e) captures the influence of the thin resistive membrane.

We note that it is necessary to discretize the flux $\left.\sigma^{\mathrm{c}} \partial_{\mathbf{n}} u\right|_{\Gamma}$ in (1e) implicitly to avoid the drastic CFL condition, as previously observed by Guyomarc'h et al. [15]. In [19], Poignard and colleagues proposed a second-order accurate finite volume method based on Cartesian grids to solve the equations in (1). However, the interface treatment they propose leads to wide stencils and large linear systems so that their approach cannot be readily applied to the simulation of a cluster with a large number of cells. The aim of this paper is to provide an efficient numerical method that makes it possible to solve the electric potential in a many-cell system. Such a numerical method is of great importance since it can help in understanding the macroscopic behavior of the potential in cell aggregates and therefore could lead the way to provide a numerical tool to compare microand meso-scale phenomena.

\subsection{Electropermeabilization model}

Electropermeabilization modeling consists in deriving a non-linear law for the surface membrane conductance $S$, or equivalently to add an electropermeabilization current in the Kirchhoff's law (1e). Generally speaking, these models describe the membrane conductance as follows:

$$
S(t, \lambda):=S_{\mathrm{L}}+S_{\mathrm{ep}}(t, \lambda)
$$

where $S_{\mathrm{L}}$ is the linear surface conductance of the membrane in the resting state and $S_{\mathrm{ep}}$ is the nonlinear conductance due to the high transmembrane voltage. In this paper, we focus on the LMSP electropermeabilization model derived by Poignard and colleagues in [20], although our numerical method can also be used for the standard model of Krassowska and colleagues [4].

\subsubsection{The LMSP model}

Leguèbe, Poignard et al. have recently proposed in [20] a new phenomenological model, which discriminates the electroporated state of the membrane and the long-lasting permeabilized state. The electroporated state of the membrane is the highly conducting state of the membrane during the pulse delivery, while the long-lasting permeabilized state is persistent after the pulse. The model reads then as:

$$
S(t, \lambda)=S_{\mathrm{L}}+S_{0} X_{0}(t, \lambda)+S_{1} X_{1}\left(t, X_{0}(t, \lambda)\right),
$$

where $S_{\mathrm{L}}, S_{0}$ and $S_{1}$ are the surface conductance of the membrane in the respective resting state, porated state and permeabilized state, and $X_{0}$ and $X_{1}$ are the degree of poration and permeabilization 
respectively, which are solutions of

$$
\left\{\begin{array}{l}
\frac{\partial X_{0}(t, \lambda)}{\partial t}=\frac{\beta_{0}(\lambda(t))-X_{0}}{\tau_{\mathrm{ep}}} \\
X_{0}(0, \lambda)=0
\end{array}\right.
$$

and

$$
\left\{\begin{array}{l}
\frac{\partial X_{1}\left(t, X_{0}\right)}{\partial t}=\max \left(\frac{\beta_{1}\left(X_{0}\right)-X_{1}}{\tau_{\text {perm }}}, \frac{\beta_{1}\left(X_{0}\right)-X_{1}}{\tau_{\text {res }}}\right), \\
X_{1}(0, \lambda)=0,
\end{array}\right.
$$

where $\beta_{0}$ and $\beta_{1}$ are even-regularized step functions

$$
\begin{array}{ll}
\text { for all } \lambda \in \mathbb{R}, & \beta_{0}(\lambda):=e^{-V_{\mathrm{ep}}^{2} / \lambda^{2}}, \\
\text { for all } X \in \mathbb{R}, & \beta_{1}(X):=e^{-X_{\mathrm{ep}}^{2} / X^{2}},
\end{array}
$$

with $V_{\mathrm{ep}}$ and $X_{\mathrm{ep}}$ are given and correspond respectively to the threshold of the membrane voltage and of the degree of poration. The coefficients $\tau_{\mathrm{ep}}, \tau_{\mathrm{perm}}$ and $\tau_{\mathrm{res}}$ are the poration characteristic time, the permeabilization characteristic time and the resealing characteristic time, respectively.

\section{Description of the computational method}

\subsection{Representation of irregular interfaces on Quad-/Oc-trees}

The irregular interface (i.e. the cell's boundary), denoted by $\Gamma$, is implicitly captured as the zero level of the level-set function $\varphi$ as first suggested by [29]. The extracellular domain $\mathscr{O}_{\mathrm{e}}$ corresponds $\varphi>0$ and the cell cytoplasm $\mathscr{O}_{\mathrm{c}}$ corresponds to $\varphi<0$.

In the case of the electropermeabilization model, the region where the solution undergoes rapid variations is focused along the cell membrane, where the discontinuities are located. Furthermore, we wish to model a large number of cells while still capturing the bulk behavior. A uniform grid would lead to a large number of points and a linear system costly to solve, we therefore choose to work with adaptive Cartesian grid of type Quad-/Oc-trees [9, 25] in order to focus the computational effort along the membrane and enable the accurate representation of a large number cells. A Quadtree is constructed by starting from a computational cell representing the entire domain and splitting it in 4 (respectively 8 if working with Octrees in three spatial dimensions) recursively. We use the following refinement criteria, proposed by Strain [32] and extended by Min [26]: split a cell $C$ if

$$
\min _{v \in \text { vertices }(\mathrm{C})}|\varphi(v)| \leq \operatorname{Lip}(\varphi) \cdot \operatorname{diag}(C)
$$

is satisfied. Here $\operatorname{Lip}(\varphi)$ is the Lipschitz constant associated to the level-set function $\varphi$, and $\operatorname{diag}(C)$ is the length of the diagonal of $C$. The process is illustrated in figure 2 . In practice, we choose $\varphi$ to be the signed distance function to the irregular interface $\Gamma$, and set $\operatorname{Lip}(\varphi)$ conservatively to 1.2. A tree is characterized by its minimum and maximum level where a level is the depth in the tree. Thus a Quadtree of level 4/6 has a coarsest resolution equivalent to a uniform grid with $16^{2}$ cells and a finest resolution equivalent to a uniform grid with $64^{2}$ cells. 

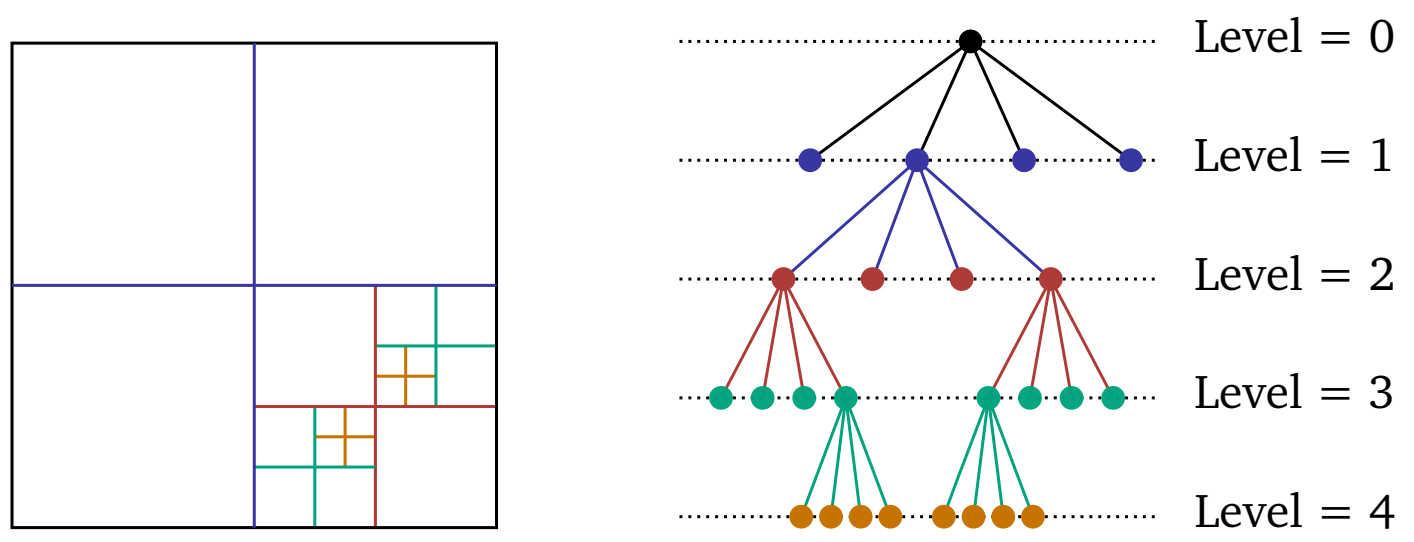

Figure 2: Illustration of a Quadtree mesh and its associated data structure.

\subsection{The Voronoi Interface Method for electropermeabilization}

The Voronoi Interface Method (VIM) introduced in [14] is designed to solve elliptic problems with discontinuities on irregular interfaces and with second-order accuracy. It consists in defining a Voronoi mesh based on an underlying Cartesian grid and solving the discontinuous elliptic problem on that new mesh. We now present an extension of the methodology for the electropermeabilization model where the discontinuity in the flux is non-trivial.

The first step consists in defining new degrees of freedom near the interface, placed along the normal to the interface, and building a corresponding Voronoi mesh. We define a Voronoi mesh as the collection of Voronoi cells such that each Voronoi cell is associated to a degree of freedom and defines the area of the computational domain that is closer to that degree of freedom than to any other. The degrees of freedom are the centers of the Quadtree cells except when the interface crosses a Quadtree cell, in which case the cell center is replaced by a pair of points on either side of the projection of the cell's center onto the interface, as illustrated in figure 3 . The procedure enforces a minimum distance between the new degrees of freedom, as explained in [14].

We now proceed to present the numerical scheme and refer to the nomenclature presented in figure 4. In order to lighten the notations, for any function $f$ defined in $\Omega$, we denote by $f^{\mathrm{e}}$ (resp. $f^{\mathrm{c}}$ ) the restriction of $f$ to $\mathscr{O}_{\mathrm{e}}\left(\right.$ resp. to $\mathscr{O}_{\mathrm{c}}$ ). We generically denote by $f_{i}, f_{j}$ and $f_{p}$ respectively the values of $f$ at the point $x_{i}, x_{j}$ and $x_{p}$ on a Voronoi mesh.

We present the derivation for $\varphi_{i}>0$ and $\varphi_{j}<0$ and start by discretizing the time evolution of the membrane voltage, $[u]$, given by equation (1e) by a standard Backward Euler scheme:

$$
C \frac{[u]^{n+1}-[u]^{n}}{\Delta t}+S^{n}[u]^{n+1}=\left(\sigma \partial_{n} u^{n+1}\right)_{\left.\right|_{\Gamma}}
$$

from which we infer:

$$
[u]^{n+1}=\frac{C[u]^{n}+\Delta t \sigma \partial_{n} u^{n+1}}{C+\Delta t S^{n}} .
$$

We then use the continuity of the flux across the interface $\Gamma$ at any time given by (1d) to write:

$$
\sigma^{\mathrm{e}} \frac{u_{p}^{\mathrm{e}}-u_{i}^{\mathrm{e}}}{d / 2}=\sigma^{\mathrm{c}} \frac{u_{j}^{\mathrm{c}}-u_{p}^{\mathrm{c}}}{d / 2}
$$



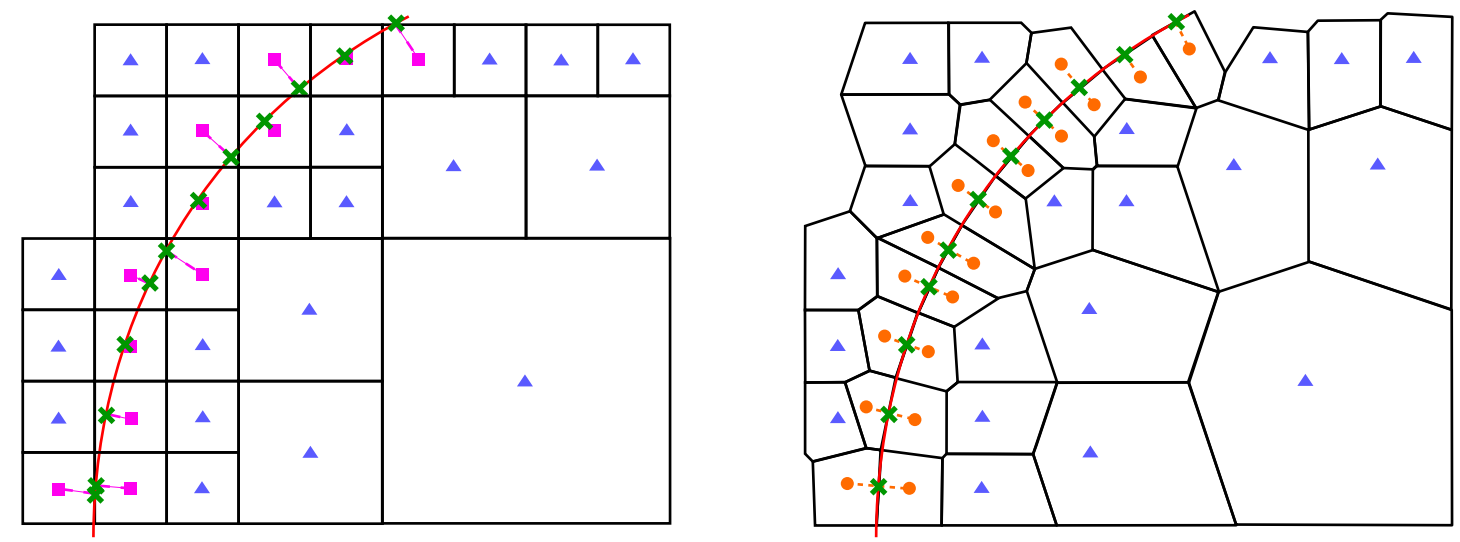

Figure 3: Illustration of the process to build a Voronoi mesh from a Quadtree grid. The cells crossed by the interface yield two new degrees of freedom on either side of the interface, avoiding the creation of points that are too close to each other. The left figure shows the original Quadtree Cartesian grid and the right figure depicts the corresponding Voronoi mesh.

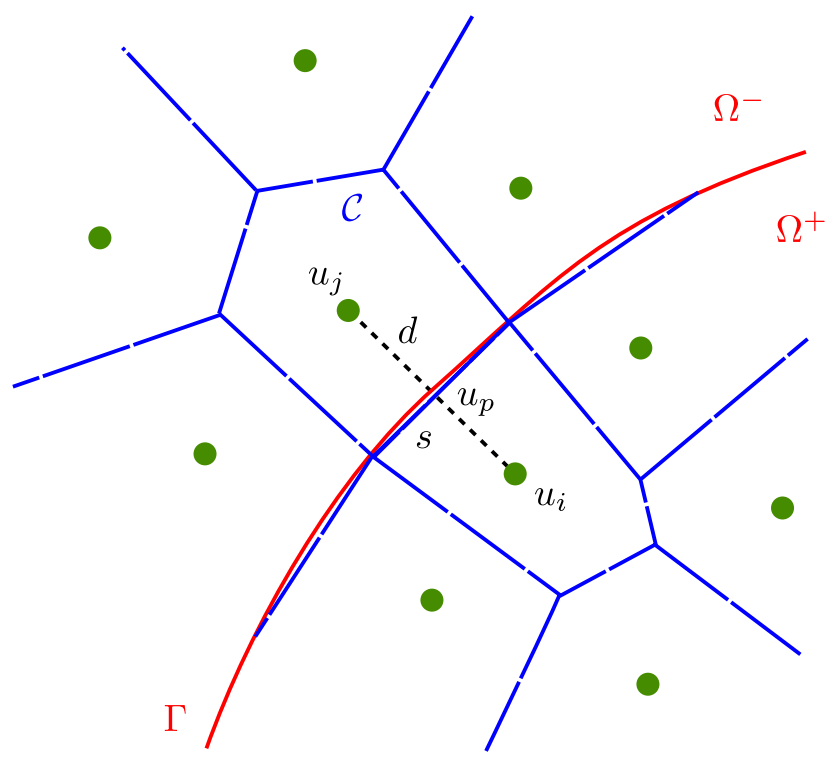

Figure 4: Nomenclature for the discretization on a Voronoi mesh. $p$ is the projection of $i$ on the interface $\Gamma$. It is also the projection of $j$ on $\Gamma$ and the halfway point between $i$ and $j$. Furthermore, $s$ is the length of the edge connecting the Voronoi cell of point $i$ and the Voronoi cell of point $j$, and $d$ is the distance between $i$ and $j$.

The discontinuity condition at the interface $u_{p}^{\mathrm{c}}=u_{p}^{\mathrm{e}}-[u]^{n+1}$ and the expression of the flux across the interface $\sigma \partial_{n} u^{n+1}=\sigma^{\mathrm{e}} \frac{u_{i}^{\mathrm{e}}-u_{p}^{\mathrm{e}}}{d / 2}$ leads to:

$$
\frac{\sigma^{\mathrm{e}}}{d / 2}\left(u_{p}^{\mathrm{e}}-u_{i}^{\mathrm{e}}\right)=\frac{\sigma^{\mathrm{c}}}{d / 2}\left(u_{j}^{\mathrm{c}}-u_{p}^{\mathrm{e}}+[u]^{n+1}\right)
$$


hence using (7) the equality

$$
\sigma^{\mathrm{e}}\left(u_{p}^{\mathrm{e}}-u_{i}^{\mathrm{e}}\right)=\sigma^{\mathrm{c}}\left(u_{j}^{\mathrm{c}}-u_{p}^{\mathrm{e}}+\frac{C[u]^{n}+\Delta t \sigma \partial_{n} u^{n+1}}{C+\Delta t S^{n}}\right)
$$

and thus

$$
\sigma^{\mathrm{e}}\left(u_{p}^{\mathrm{e}}-u_{i}^{\mathrm{e}}\right)=\sigma^{\mathrm{c}}\left(u_{j}^{\mathrm{c}}-u_{p}^{\mathrm{e}}+\frac{C[u]^{n}}{C+\Delta t S^{n}}+\frac{\Delta t}{C+\Delta t S^{n}} \sigma^{\mathrm{e}} \frac{u_{i}^{\mathrm{e}}-u_{p}^{\mathrm{e}}}{d / 2}\right),
$$

which leads to the expression of $u_{p}^{\mathrm{e}}$ :

$$
u_{p}^{\mathrm{e}}=\left(\sigma^{\mathrm{e}} u_{i}^{\mathrm{e}}+\sigma^{\mathrm{c}} u_{j}^{\mathrm{c}}+\frac{\sigma^{\mathrm{c}} C[u]^{n}}{C+\Delta t S^{n}}+\frac{\sigma^{\mathrm{e}} \sigma^{\mathrm{c}} \Delta t}{\left(C+\Delta t S^{n}\right) d / 2} u_{i}^{\mathrm{e}}\right) /\left(\sigma^{\mathrm{e}}+\sigma^{\mathrm{c}}+\frac{\sigma^{\mathrm{e}} \sigma^{\mathrm{c}} \Delta t}{\left(C+\Delta t S^{n}\right) d / 2}\right) .
$$

We can then use $u_{p}^{\mathrm{e}}$ into the discretization for the Laplacian equation on the Voronoi cells. More precisely, integrating Laplace equation on the Voronoi cell leads to

$$
\sum_{k \in\{\partial \mathscr{C} \backslash \Gamma\}} s_{k} \sigma^{\mathrm{e}} \frac{u_{k}^{\mathrm{e}}-u_{i}^{\mathrm{e}}}{d_{k}}+\sigma^{\mathrm{e}} s \frac{u_{p}^{\mathrm{e}}-u_{i}^{\mathrm{e}}}{d / 2}=0 .
$$

In the above equality, the first sum represents the contribution from the faces of the Voronoi cell where the neighbor point $u_{k}$ is in the same domain, and the second term captures the interface contribution. We then get the following:

$\sum_{k \in\{\partial \mathscr{C} \backslash \Gamma\}} s_{k} \sigma^{\mathrm{e}} \frac{u_{k}^{\mathrm{e}}-u_{i}^{\mathrm{e}}}{d_{k}}+s \frac{\sigma^{\mathrm{e}} \sigma^{\mathrm{c}}}{\sigma^{\mathrm{e}}+\sigma^{\mathrm{c}}+\frac{\sigma^{\mathrm{e}} \sigma^{\mathrm{c}} \Delta t}{\left(C+\Delta t S^{n}\right) d / 2}} \frac{u_{j}^{\mathrm{c}}-u_{i}^{\mathrm{e}}}{d / 2}=-s \frac{\sigma^{\mathrm{e}} \sigma^{\mathrm{c}}}{\sigma^{\mathrm{e}}+\sigma^{\mathrm{c}}+\frac{\sigma^{\mathrm{e}} \sigma^{\mathrm{c}} \Delta t}{\left(C+\Delta t S^{n}\right) d / 2}} \frac{C[u]^{n}}{\left(C+\Delta t S^{n}\right) d / 2}$.

Similarly, we obtain the final expression for the case when $\varphi_{i}<0$ and $\varphi_{j}>0$ :

$$
\sum_{k \in\{\partial \mathscr{C} \backslash \Gamma\}} s_{k} \sigma^{\mathrm{e}} \frac{u_{k}^{\mathrm{e}}-u_{i}^{\mathrm{e}}}{d_{k}}+s \hat{\sigma} \frac{u_{j}-u_{i}}{d / 2}=\operatorname{sgn}\left(\varphi_{i}\right) s \hat{\sigma} \frac{C[u]^{n}}{\left(C+\Delta t S^{n}\right) d / 2},
$$

with

$$
\hat{\sigma}=\frac{\sigma^{\mathrm{e}} \sigma^{\mathrm{c}}}{\sigma^{\mathrm{e}}+\sigma^{\mathrm{c}}+\frac{\sigma^{\mathrm{e}} \sigma^{\mathrm{c}} \Delta t}{\left(C+\Delta t S^{n}\right) d / 2}} .
$$

The points far from the interface are treated with a classical finite volume discretization on the Voronoi partition. Since all the coefficients involved in $\hat{\sigma}$ are positive and the discontinuity $[u]^{n}$ appears only in the right hand side, this produces a symmetric positive definite linear system.

Remark 1 (Formula for BDF2). The numerical simulations of Section 4 only deal with standard Backward Euler scheme, which is accurate enough for our problem. However second-order Backward Differentiation (BDF2) may be needed for the time-discretization. The above expression has then to be replaced by

$$
\sum_{k \in\{\partial \mathscr{C} \backslash \Gamma\}} s_{k} \sigma^{\mathrm{e}} \frac{u_{k}^{\mathrm{e}}-u_{i}^{\mathrm{e}}}{d_{k}}+s \tilde{\sigma} \frac{u_{j}-u_{i}}{d / 2}=\operatorname{sgn}\left(\varphi_{i}\right) s \tilde{\sigma} \frac{C\left(2[u]^{n}-\frac{1}{2}[u]^{n-1}\right)}{\left(\frac{3}{2} C+\Delta t S^{n}\right) d / 2},
$$


with

$$
\tilde{\sigma}=\frac{\sigma^{\mathrm{e}} \sigma^{\mathrm{c}}}{\sigma^{\mathrm{e}}+\sigma^{\mathrm{c}}+\frac{\sigma^{\mathrm{e}} \sigma^{\mathrm{c}} \Delta t}{\left(\frac{3}{2} C+\Delta t S^{n}\right) d / 2}} .
$$

We next present numerical results that show the accuracy of our method in section 4 . We then study numerically the permeabilization of cell aggregates in 3D configurations in section 5 .

\section{Numerical results}

Throughout this section, the parameters described in table 1 are used. All the computations are performed with double precision.

\begin{tabular}{llll}
\hline Variable & Symbol & Value & Unit \\
\hline Extracellular conductivity & $\sigma^{\mathrm{e}}$ & 15 & $\mathrm{~S} / \mathrm{m}$ \\
Intracellular conductivity & $\sigma^{\mathrm{c}}$ & 1 & $\mathrm{~S} / \mathrm{m}$ \\
Capacitance & $C$ & $9.5 \cdot 10^{-3}$ & $\mathrm{~F} / \mathrm{m}^{2}$ \\
Membrane surface conductivity & $S_{L}$ & 1.9 & $\mathrm{~S} / \mathrm{m}^{2}$ \\
Cell radius & $R_{1}$ & 50 & $\mu \mathrm{m}$ \\
Voltage threshold for poration & $V_{e p}$ & $258 \cdot 10^{-3}$ & $\mathrm{~V}$ \\
Threshold for poration degree & $X_{e p}$ & 0.5 & - \\
Poration characteristic time & $\tau_{e p}$ & $10^{-6}$ & $\mathrm{~S}$ \\
Permeabilization characteristic time & $\tau_{p e r m}$ & $10^{-6}$ & $\mathrm{~S}$ \\
Resealing characteristic time & $\tau_{r e s}$ & 60 & $\mathrm{~S}$ \\
Porated membrane conductance & $S_{0}$ & $1.1 \cdot 10^{6}$ & $\mathrm{~S} / \mathrm{m}^{2}$ \\
Permeabilized membrane conductance & $S_{1}$ & $10^{4}$ & $\mathrm{~S} / \mathrm{m}^{2}$ \\
\hline
\end{tabular}

Table 1: Physical and computational parameters used for the simulations.

\subsection{Solution to the static linear problem in two spatial dimensions}

Consider the static linear problem, i.e. $C=0$ in equation (1e), in the case where the cell is a disk of radius $R_{1}$, embedded in a concentric bath of radius $R_{2}$. Assume that the electric potential

at $R_{2}$ is $u_{\mid R_{2}}=\frac{1}{2} g \cos \theta$, with $g=R_{2} E$. In practice, we choose $E=40 \mathrm{kV} / \mathrm{m}, R_{1}=50 \mu \mathrm{m}$ and $R_{2}=0.6 \mathrm{~mm}$. Then the exact solution of the static problem is explicitly given by:

$$
\begin{aligned}
u^{\mathrm{e}} & =\left(\alpha^{\mathrm{e}} r+\frac{\beta^{\mathrm{e}}}{r}\right) \cos (\theta), \\
u^{\mathrm{c}} & =\alpha^{\mathrm{c}} r \cos (\theta),
\end{aligned}
$$


where the coefficients $\alpha^{\mathrm{c}}, \alpha^{\mathrm{e}}$ and $\beta^{\mathrm{e}}$ are given by

$$
\begin{aligned}
\alpha^{\mathrm{c}} & =\left(\left(\frac{\sigma^{\mathrm{c}}}{S_{L} R_{1}}+1+\frac{\sigma^{\mathrm{c}}}{\sigma^{\mathrm{e}}}\right) R_{2}+\left(\frac{\sigma^{\mathrm{c}}}{S_{L} R_{1}}+1-\frac{\sigma^{\mathrm{c}}}{\sigma^{\mathrm{e}}}\right) \frac{R_{1}^{2}}{R_{2}}\right)^{-1} g \\
\alpha^{\mathrm{e}} & =\frac{1}{2}\left(\frac{\sigma^{\mathrm{c}}}{S_{L} R_{1}}+1+\frac{\sigma^{\mathrm{c}}}{\sigma^{\mathrm{e}}}\right) \alpha^{\mathrm{c}}, \\
\beta^{\mathrm{e}} & =\frac{1}{2}\left(\frac{\sigma^{\mathrm{c}}}{S_{L} R_{1}}+1-\frac{\sigma^{\mathrm{c}}}{\sigma^{\mathrm{e}}}\right) \alpha^{\mathrm{c}} R_{1}^{2}
\end{aligned}
$$

from which we infer the static membrane voltage:

$$
[u]=\frac{\sigma^{\mathrm{c}}}{S_{L}} \alpha^{\mathrm{c}} \cos (\theta)
$$

For the numerical tests, we choose a computational domain entirely contained in the larger circle and we project the explicit solution $u_{e}$ given by (8) on the boundary of the simulation box in order to impose the appropriate Dirichlet boundary condition on our numerical solution.

Note that this problem is time independent and discretized implicitly, and is therefore a Poisson equation with a jump condition on the irregular interface, which is solved by a single iteration of the solver. Figure 5 presents a visualization of the computed electric potential and the computed membrane voltage. We monitor the convergence of the solver on the membrane voltage and report the results in table 2. The computed error corresponds to the largest error over the membrane between the exact membrane potential and the computed membrane potential. The convergence results indicate that second-order accuracy is achieved.

\begin{tabular}{|c|c|c|c|c|}
\hline level $(\min / \mathrm{max})$ & $L^{\infty}$ error on $u$ & order & $L^{\infty}$ error on $[u]$ & order \\
\hline $3 / 5$ & $3.76 \cdot 10^{-6}$ & - & $3.95 \cdot 10^{-6}$ & - \\
$4 / 6$ & $1.98 \cdot 10^{-6}$ & 0.93 & $1.98 \cdot 10^{-6}$ & 1.00 \\
$5 / 7$ & $7.84 \cdot 10^{-7}$ & 1.34 & $7.75 \cdot 10^{-7}$ & 1.35 \\
$6 / 8$ & $2.56 \cdot 10^{-7}$ & 1.62 & $2.52 \cdot 10^{-7}$ & 1.62 \\
$7 / 9$ & $7.33 \cdot 10^{-8}$ & 1.80 & $7.20 \cdot 10^{-8}$ & 1.81 \\
$8 / 10$ & $1.98 \cdot 10^{-8}$ & 1.89 & $1.91 \cdot 10^{-8}$ & 1.91 \\
$9 / 11$ & $5.35 \cdot 10^{-9}$ & 1.89 & $5.11 \cdot 10^{-9}$ & 1.90 \\
\hline
\end{tabular}

Table 2: Convergence of the solver for the static case of subsection4.1.

\subsection{Dynamic solution in two spatial dimensions}

We now study the convergence of our method for the dynamic solution to the time-dependent linear problem in the two spatial dimensions case of a circular single cell. The geometrical framework is similar to that of section 4.1. We focus on the membrane voltage, which is the biophysically 

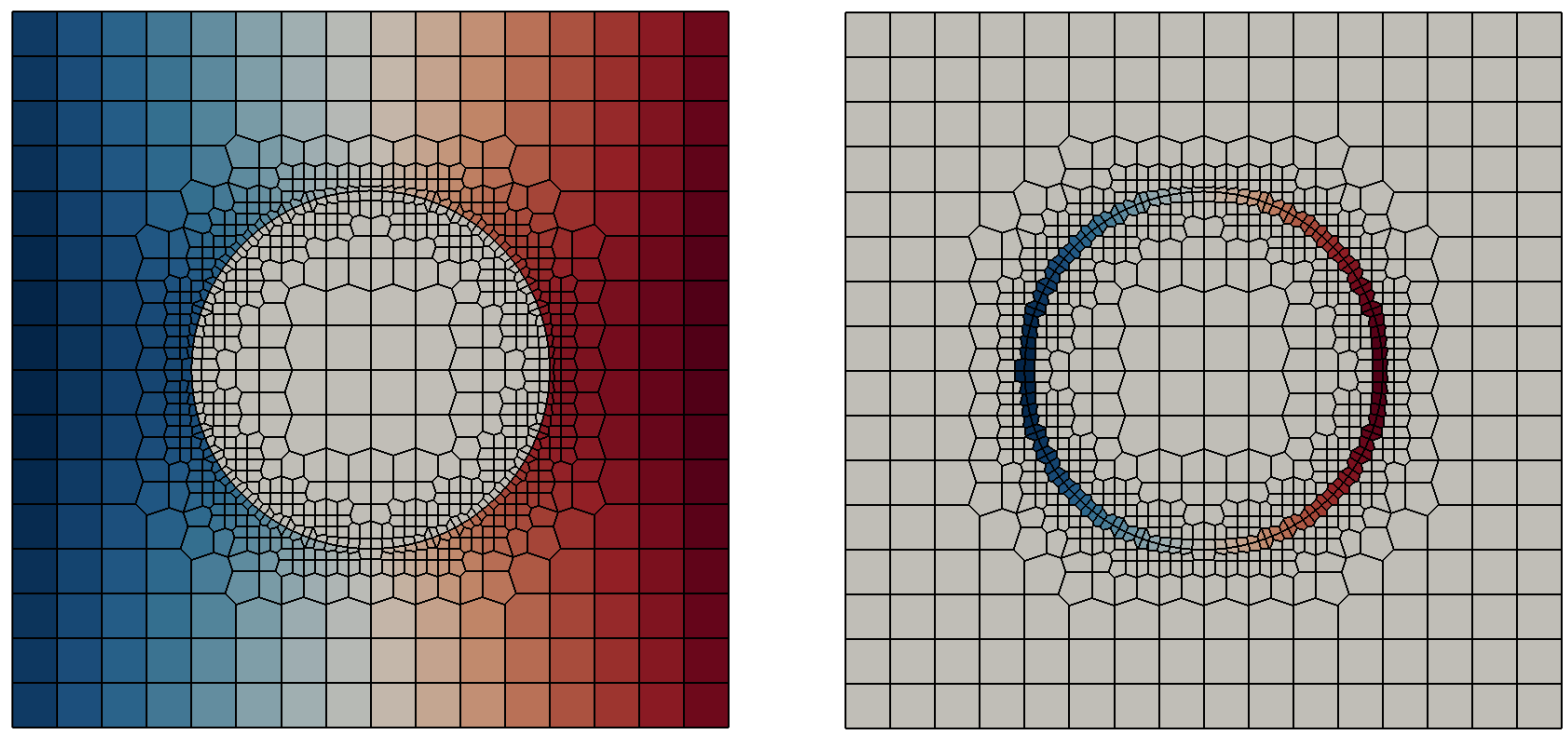

Figure 5: Representation of the numerical potential (left) and of the membrane voltage (right). These functions are approximations of the potential $u$ and the membrane voltage $[u]$ given by equations (8) and (9), respectively.

relevant quantity. Applying a time-dependent Dirichlet condition $(t, \theta): \rightarrow g(t) \cos \theta$ on the outer boundary, we infer that the membrane voltage satisfies the ordinary differential equation

$$
\partial_{t}[u]+\frac{S_{L}-B}{C}[u]=\frac{A}{C} g \cos (\theta),
$$

with

$$
A=2 \sigma^{\mathrm{c}} \sigma^{\mathrm{e}} R_{2} K, \quad B=-\sigma^{\mathrm{c}} \sigma^{\mathrm{e}}\left(R_{1}+R_{2}^{2} / R_{1}\right) K \quad \text { where } \quad K=\frac{1}{R_{1}^{2}\left(\sigma^{\mathrm{e}}-\sigma^{\mathrm{c}}\right)+R_{2}^{2}\left(\sigma^{\mathrm{e}}+\sigma^{\mathrm{c}}\right)} .
$$

Given that the initial discontinuity is $[u]_{t=0}=0$, we obtain:

$$
[u](t, \theta)=\frac{A}{S_{L}-B} g \cos (\theta)\left(1-e^{-\frac{S_{L}-B}{C} t}\right) .
$$

The solution and the membrane potential are represented in figure 6 . We choose the time step as $\Delta t=\Delta x_{\min } / 40$ and solve the problem until the final time $t_{f}=1 \mu \mathrm{s}$. The convergence of the solver on the membrane potential $[u]$ is presented in table 3 and figures 6 and 7 . The error is computed as the maximum error over the entire membrane between the calculated membrane potential $[u]$ and the expected exact value. The results indicate an order of accuracy of about 1.5.

\subsection{Validation with the static linear case in three spatial dimensions}

In spherical coordinates, a possible form of the solution to the homogeneous Laplace equation that is independent of the azimuthal angle $\varphi$ is

$$
f(r, \theta, \varphi)=f(r, \theta)=\sum_{l=0}^{\infty}\left(A_{l} r^{l}+\frac{B_{l}}{r^{l+1}}\right) P_{l}(\cos (\theta)),
$$



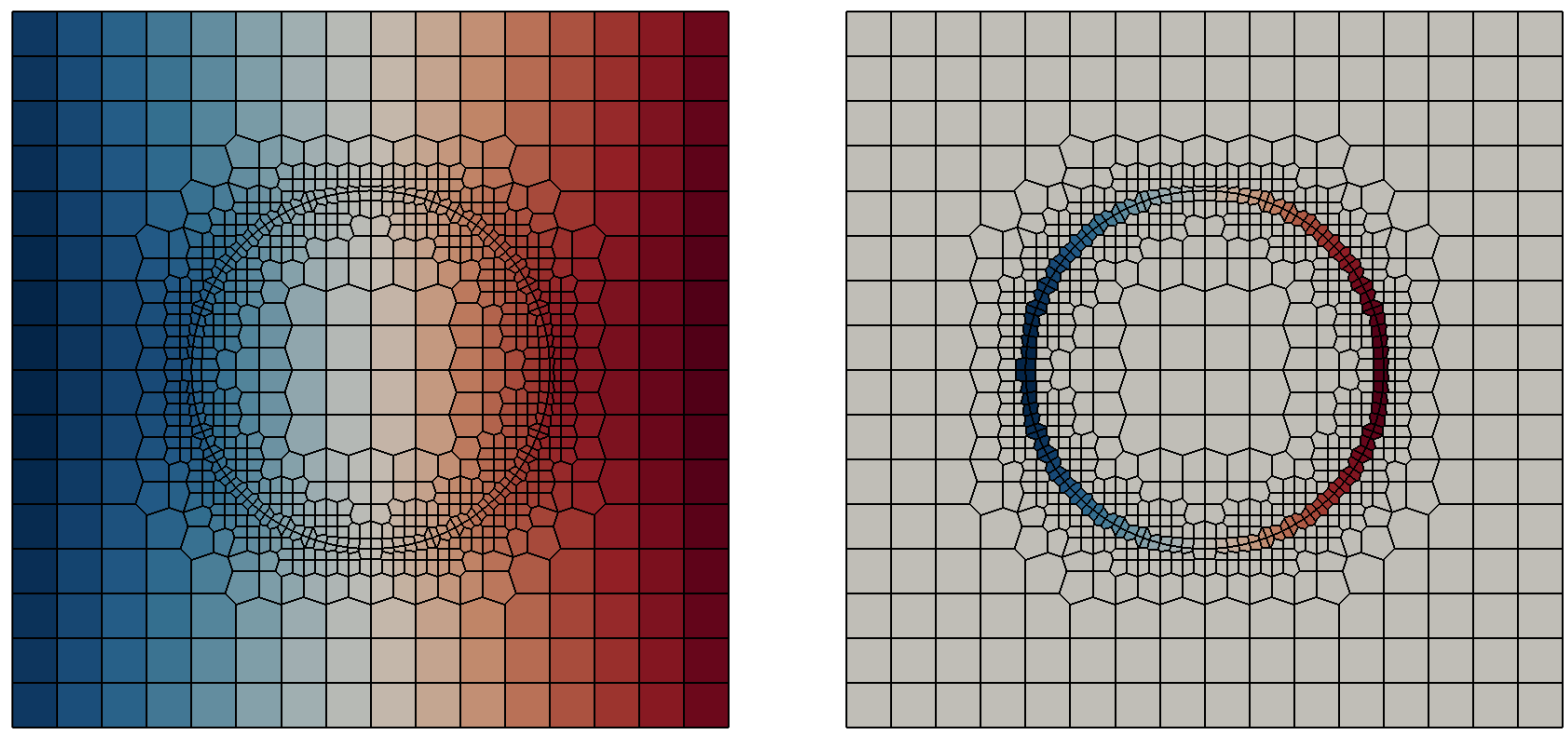

Figure 6: Representation of the electric potential and the transmembrane voltage given by equation (1), in the bidimensional circular framework of section 4.2.

\begin{tabular}{|c|c|c|c|c|}
\cline { 2 - 5 } \multicolumn{1}{c|}{} & \multicolumn{2}{c|}{ first order time } & \multicolumn{2}{c|}{ second-order time } \\
\hline level (min/max) & error $\left(L^{\infty}\right)$ & order & error $\left(L^{\infty}\right)$ & order \\
\hline $3 / 5$ & $2.58 \cdot 10^{-5}$ & - & $3.29 \cdot 10^{-6}$ & - \\
$4 / 6$ & $1.26 \cdot 10^{-5}$ & 1.03 & $2.75 \cdot 10^{-6}$ & 0.26 \\
$5 / 7$ & $5.91 \cdot 10^{-6}$ & 1.09 & $1.18 \cdot 10^{-6}$ & 1.22 \\
$6 / 8$ & $2.76 \cdot 10^{-6}$ & 1.10 & $3.98 \cdot 10^{-7}$ & 1.57 \\
$7 / 9$ & $1.30 \cdot 10^{-6}$ & 1.08 & $1.19 \cdot 10^{-7}$ & 1.74 \\
$8 / 10$ & $6.30 \cdot 10^{-7}$ & 1.05 & $3.91 \cdot 10^{-8}$ & 1.61 \\
$9 / 11$ & $3.09 \cdot 10^{-7}$ & 1.03 & $1.33 \cdot 10^{-8}$ & 1.56 \\
\hline
\end{tabular}

Table 3: Error on the membrane electric potential discontinuity $[u]$ in the dynamic case 4.2 after $t_{f}=1 \mu s$, with $E=40 \mathrm{kV} / \mathrm{m}$ and $\Delta t=\Delta x_{\min } / 40$, using the second order time discretization.

where $P_{l}$ is the $l^{\text {th }}$ Legendre polynomial and $\theta$ is the angle with the $z$-axis, $\theta=\tan ^{-1}\left(\sqrt{x^{2}+y^{2}} / z\right)$. For instance $P_{1}(x)=x$. We then choose:

$$
U^{\mathrm{e}}=\left(\alpha^{\mathrm{e}} r+\frac{\beta^{\mathrm{e}}}{r^{2}}\right) \cos (\theta) \quad \text { and } \quad U^{\mathrm{c}}=\alpha^{\mathrm{c}} r \cos (\theta) .
$$



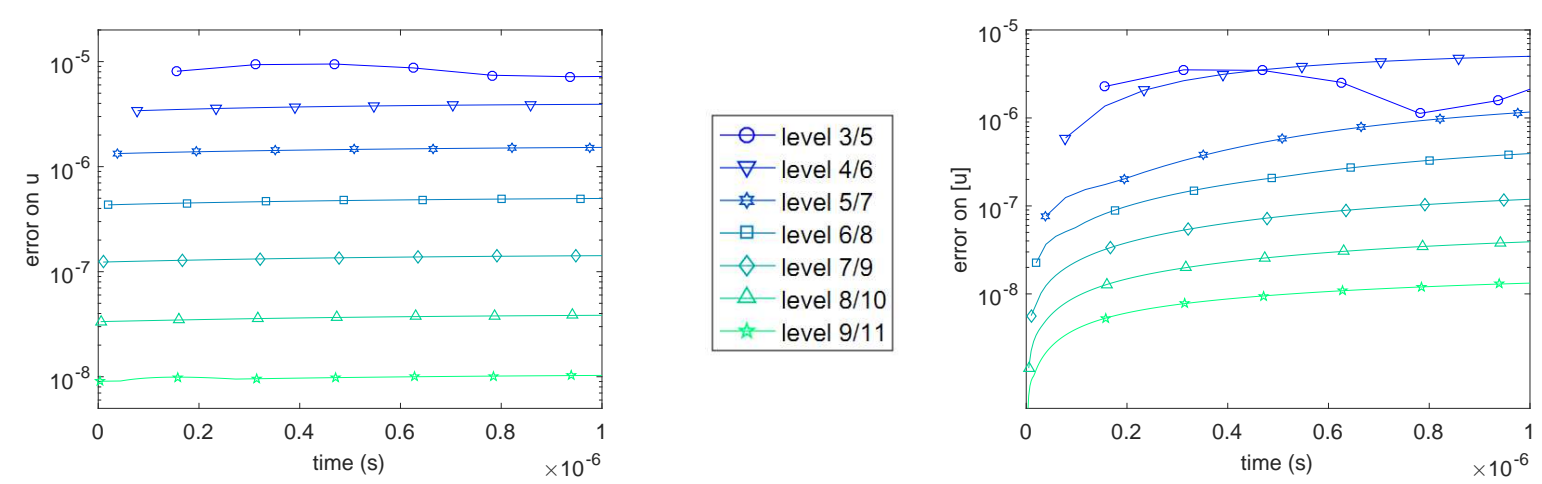

Figure 7: Visualization of the $L^{\infty}$ error on the electric potential $u$ (left) and on the membrane discontinuity $[u]$ (right) as a function of time for the dynamic analytical example 4.2, and for grid resolutions $3 / 5$ (top curves) through $9 / 11$ (bottom curves).

Matching the boundary conditions for a unidirectional external potential of magnitude $g=E R_{2}$ and a cell of radius $R_{1}$, from

$$
\left\{\begin{array} { l } 
{ u ^ { \mathrm { e } } ( R _ { 2 } ) = g } \\
{ [ \sigma \partial _ { n } u ] _ { | R _ { 1 } } = 0 \quad , \text { we infer: } } \\
{ [ u ] _ { | R _ { 1 } } = U ^ { \mathrm { e } } - U ^ { \mathrm { c } } }
\end{array} \left\{\begin{array}{l}
\alpha^{\mathrm{e}}=R_{2}^{2}\left(\sigma^{\mathrm{c}}+2 \sigma^{\mathrm{e}}\right) K g-R_{1}^{2} \sigma^{\mathrm{c}} K \frac{[u]}{\cos (\theta)} \\
\beta^{\mathrm{e}}=R_{1}^{3} R_{2}^{2}\left(\sigma^{\mathrm{e}}-\sigma^{\mathrm{c}}\right) K g+R_{1}^{2} R_{2}^{3} \sigma^{\mathrm{c}} K \frac{[u]}{\cos (\theta)} \\
\alpha^{\mathrm{c}}=3 \sigma^{\mathrm{e}} R_{2}^{2} K g-\sigma^{\mathrm{e}}\left(R_{1}^{2}+2 \frac{R_{2}^{3}}{R_{1}}\right) K \frac{[u]}{\cos (\theta)}
\end{array}\right.\right.
$$

with $K^{-1}=R_{1}^{3}\left(\sigma^{\mathrm{e}}-\sigma^{\mathrm{c}}\right)+R_{2}^{3}\left(2 \sigma^{\mathrm{e}}+\sigma^{\mathrm{c}}\right)$. Since we are working with the static linear case (i.e. $\left.S=S_{L}\right),[u]$ is given by:

$$
[u]=\frac{A}{S_{L}-B} g \cos \theta, \quad \text { with } \quad A=3 \sigma^{\mathrm{c}} \sigma^{\mathrm{e}} R_{2}^{2} K \quad \text { and } \quad B=-\sigma^{\mathrm{c}} \sigma^{\mathrm{e}}\left(R_{1}^{2}+2 \frac{R_{2}^{3}}{R_{1}}\right) K .
$$

We monitor the convergence of the solver for this exact solution with $E=10 \mathrm{kV} / \mathrm{m}, \Omega=\left[-10^{-4}, 10^{-4}\right]^{3}$, $R_{1}=5 \cdot 10^{-6}$ and $R_{2}=6 \cdot 10^{-4}$. The results are presented in table 4 and seem to an order of accuracy slightly above one, though the asymptotic regime is not reached yet. Unfortunately, larger simulations are not practical with the current framework and would require a parallel environment, to which we plan to extend our method in the future.

\subsection{Validation with the dynamic linear case in three spatial dimensions}

For this validation, we use the time-independent data $g$ as in the previous section. However, $[u]$ now satisfies the dynamic equation given, for a constant $S=S_{L}$, as:

$$
C \partial_{t}[u]+S_{L}[u]=\sigma^{\mathrm{c}} \partial_{n} U^{\mathrm{c}}
$$




\begin{tabular}{|c|c|c|c|c|}
\cline { 2 - 5 } \multicolumn{1}{c|}{} & \multicolumn{2}{c|}{ potential $U$} & \multicolumn{2}{c|}{ Membrane potential $[u]$} \\
\hline level $(\mathrm{min} / \mathrm{max})$ & error $\left(L^{\infty}\right)$ & order & error $\left(L^{\infty}\right)$ & order \\
\hline $3 / 5$ & $4.14 \cdot 10^{-6}$ & - & $5.71 \cdot 10^{-6}$ & - \\
$4 / 6$ & $3.72 \cdot 10^{-6}$ & 0.15 & $3.80 \cdot 10^{-6}$ & 0.59 \\
$5 / 7$ & $2.01 \cdot 10^{-6}$ & 0.89 & $1.87 \cdot 10^{-6}$ & 1.02 \\
$6 / 8$ & $7.49 \cdot 10^{-7}$ & 1.42 & $7.14 \cdot 10^{-7}$ & 1.39 \\
$7 / 9$ & $2.43 \cdot 10^{-7}$ & 1.62 & $2.32 \cdot 10^{-7}$ & 1.62 \\
\hline
\end{tabular}

Table 4: Convergence of the solver for the linear static case in three spatial dimensions 4.3.

hence

$$
\partial_{t}[u]+\frac{S_{L}-B}{C}[u]=\frac{A}{C} g \cos (\theta),
$$

where $A$ and $B$ are given by equation (10). Given that the initial discontinuity is $[u]_{t=0}=0$, we obtain:

$$
[u](t, \theta)=\frac{A}{S_{L}-B} g \cos (\theta)\left(1-e^{-\frac{S_{L}-B}{C} t}\right) .
$$

We monitor the convergence of the solver for this exact solution with $E=40 \mathrm{kV} / \mathrm{m}, \Omega=$ $\left[-10^{-4}, 10^{-4}\right]^{3}, R_{1}=5 \cdot 10^{-5}, R_{2}=6 \cdot 10^{-4}, t_{f}=10^{-6} \mathrm{~s}$ and $\Delta t=\Delta x_{\min } / 40$. The results are presented in table 5 and in figures 8 and 9 . We observe first order convergence when using the first order time discretization. However, the second order time discretization does not produce second order convergence. It is possible that this is due to the non-linearity of the problem. The errors resulting from the second order time discretization are almost an order of magnitude smaller than the ones observed for the first order time discretization.
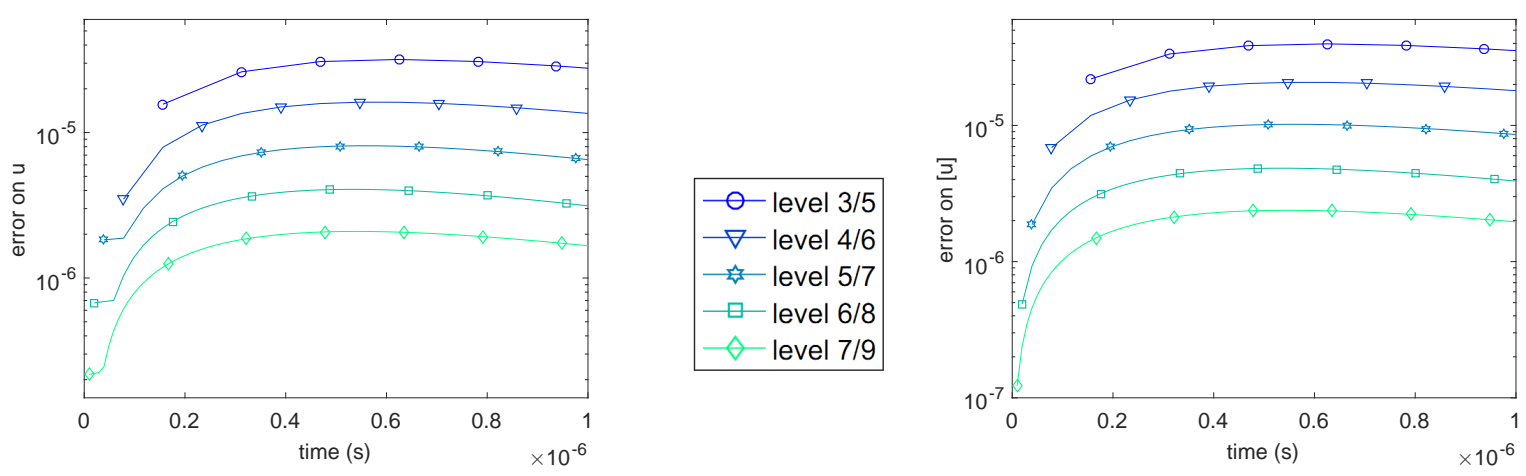

Figure 8: Visualization of the $L^{\infty}$ error on the electric potential $u$ (left) and on the membrane discontinuity $[u]$ (right) as a function of time for the dynamic analytical example 4.4, for grid resolutions $3 / 5$ through $7 / 9$, using the first order discretization in time. 
First order time discretization

\begin{tabular}{|c|c|c|c|c|}
\cline { 2 - 5 } \multicolumn{1}{c|}{} & \multicolumn{2}{c|}{ potential $U$} & \multicolumn{2}{c|}{ Membrane potential $[u]$} \\
\hline level $(\min / \max )$ & error $\left(L^{\infty}\right)$ & order & error $\left(L^{\infty}\right)$ & order \\
\hline $3 / 5$ & $2.62 \cdot 10^{-5}$ & - & $3.38 \cdot 10^{-5}$ & - \\
$4 / 6$ & $1.34 \cdot 10^{-5}$ & 0.93 & $1.78 \cdot 10^{-5}$ & 1.10 \\
$5 / 7$ & $6.44 \cdot 10^{-6}$ & 1.06 & $8.49 \cdot 10^{-6}$ & 1.07 \\
$6 / 8$ & $3.09 \cdot 10^{-6}$ & 1.06 & $3.86 \cdot 10^{-6}$ & 1.14 \\
$7 / 9$ & $1.66 \cdot 10^{-6}$ & 0.90 & $1.95 \cdot 10^{-6}$ & 0.99 \\
\hline
\end{tabular}

Second order time discretization

\begin{tabular}{|c|c|c|c|c|}
\cline { 2 - 5 } \multicolumn{1}{c|}{} & \multicolumn{2}{c|}{ potential $U$} & \multicolumn{2}{c|}{ Membrane potential $[u]$} \\
\hline level $(\min / \max )$ & error $\left(L^{\infty}\right)$ & order & error $\left(L^{\infty}\right)$ & order \\
\hline $3 / 5$ & $4.16 \cdot 10^{-6}$ & - & $9.99 \cdot 10^{-6}$ & - \\
$4 / 6$ & $3.71 \cdot 10^{-6}$ & 0.17 & $5.63 \cdot 10^{-6}$ & 0.83 \\
$5 / 7$ & $1.99 \cdot 10^{-6}$ & 0.90 & $2.46 \cdot 10^{-6}$ & 1.19 \\
$6 / 8$ & $7.41 \cdot 10^{-7}$ & 1.43 & $8.24 \cdot 10^{-7}$ & 1.58 \\
$7 / 9$ & $3.39 \cdot 10^{-7}$ & 1.13 & $6.11 \cdot 10^{-7}$ & 0.43 \\
\hline
\end{tabular}

Table 5: Convergence of the solver for the linear dynamic case in three spatial dimensions 4.4 for $E=40 \mathrm{kV} / \mathrm{m}$ and $\Delta t=\Delta x_{\min } / 40$ at the final time $t_{f}=1 \mu \mathrm{s}$ using the first order time discretization (top) and second order time discretization (bottom).
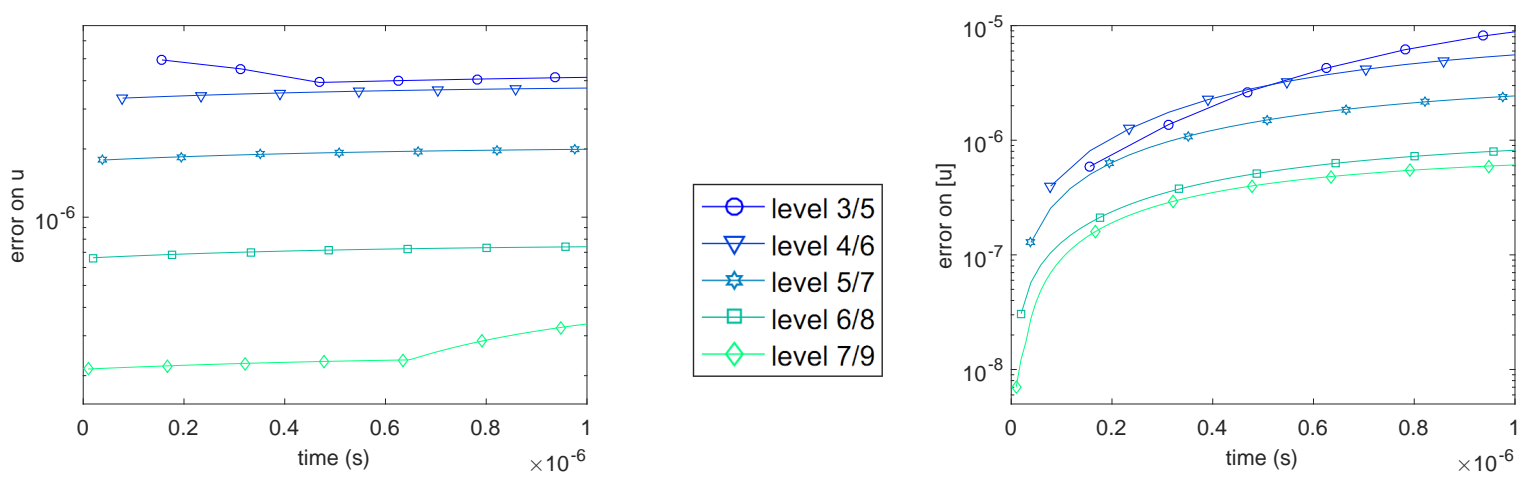

Figure 9: Visualization of the $L^{\infty}$ error on the electric potential $u$ (left) and on the membrane discontinuity $[u]$ (right) as a function of time for the dynamic analytical example 4.4, for grid resolutions $3 / 5$ through $7 / 9$, using the second order discretization in time.

\subsection{Convergence in time and space for the non-linear model in a single cell}

We propose to monitor the convergence of the solver in time and space for the full non-linear model. We consider a spherical cell with radius $r_{0}=50 \mu \mathrm{m}$, centered in a box of length $4 r_{0}$. We apply an electric field in the $z$-direction with intensity $E=40 \mathrm{kV} / \mathrm{m}$ and compute the solution at a final time of $t_{f}=1.5 \cdot 10^{-6} \mathrm{~s}$. 
Starting with the convergence in space, we solve the problem for increasing spatial resolutions and with a fixed time step $\Delta t=9.77 \cdot 10^{-9} \mathrm{~s}$. Starting with a mesh of resolution $3 / 6$, i.e. with finest resolution equivalent to $64^{3}$ cells, we increase the maximum resolution up to 9 , i.e. equivalent to $512^{3}$. The electric discontinuity $[u]$ at the pole of the cell is monitored in figure 10 and we observe convergence towards a solution.
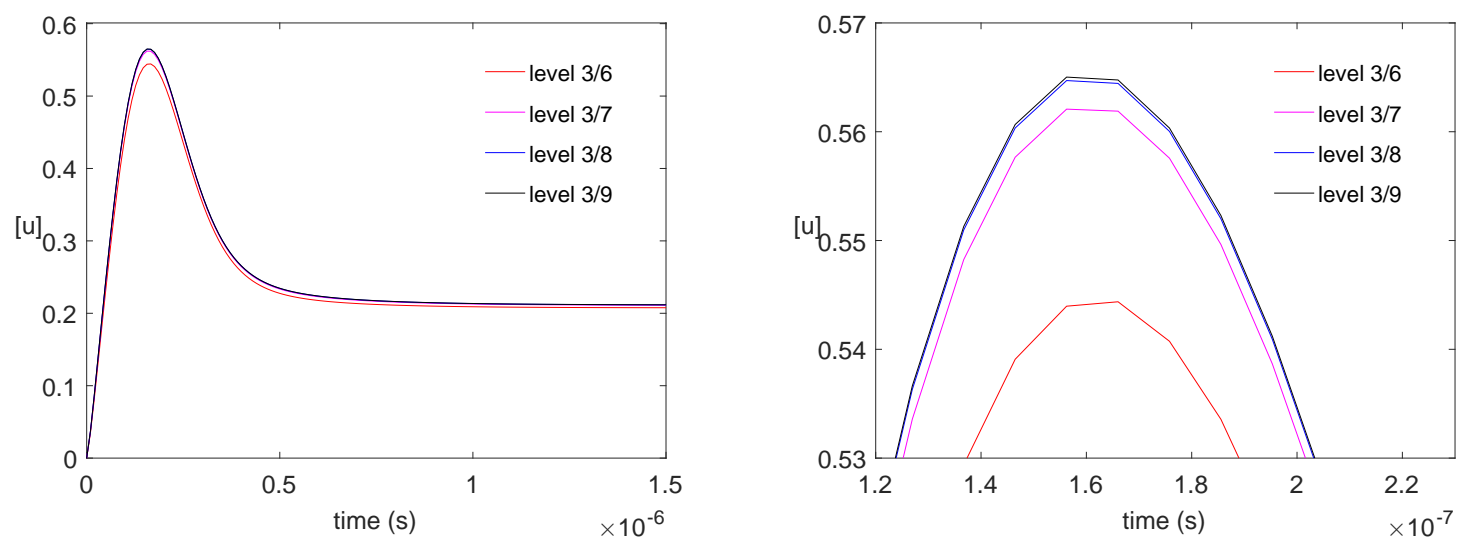

Figure 10: Convergence of the solver in space for the non-linear dynamic case with a single cell. The time step $\Delta t=9.77 \cdot 10^{-9} \mathrm{~s}$ is kept constant while the spatial resolution is increased. We observe convergence.

Next, we observe the convergence of the solver for a fixed space resolution of $3 / 7$ as the time step is halved successively. Again, we monitor the electric discontinuity $[u]$ at the pole of the cell and the results are presented in figure 11 together with the finest resolution from the previous spatial convergence study. The solver converges, and furthermore we observe similar accuracy with a resolution 3/7 than for the fine resolution 3/9 for the same time step.

These results are consistent with the model which is highly non-linear in time but well-behaved in space. Therefore, as long as the geometry is correctly resolved, the spatial accuracy is dominated by the temporal errors and a finer time step is more critical than a fine spatial resolution.

\section{Computational study of the permeabilization of three dimensional cell arrays}

The combination of the sharp treatment of the interfacial jump condition, the symmetry and definite positiveness of the linear system, with the adaptive Cartesian grid, enables the study of electroporation beyond a single cell. In what follows, we present electroporation simulations on arrays of cells and study the influence of cells' geometry and the shadowing effects.

\subsection{Shadowing effect}

We study the behavior of the solver for a $3 \times 3 \times 3$ array of spherical cells with radii $r_{0}=5 \mu \mathrm{m}$, located periodically in an inner box of $1 \mathrm{~cm}^{3}$. In order to ensure that the entire aggregate is embedded in an homogeneous electric field, we place it at the center of a $2 \mathrm{~cm}^{3}$ computational domain as illustrated in the left part of figure 13. We take a time step small enough to capture the non-linear 

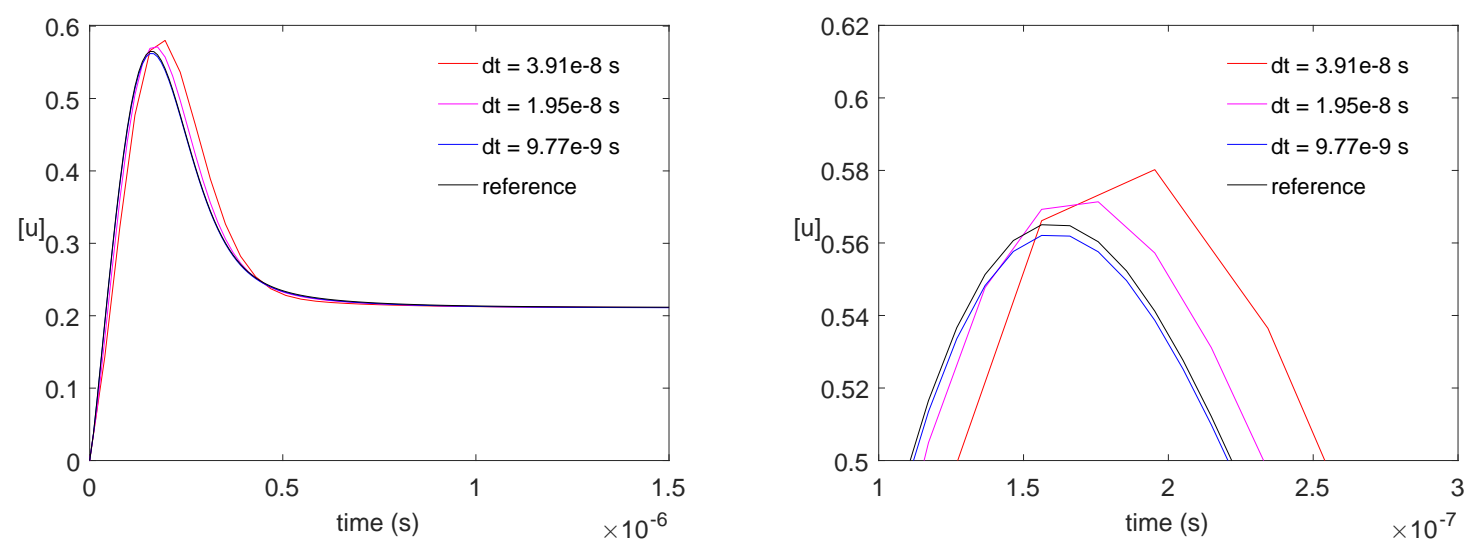

Figure 11: Convergence of the solver in time for the non-linear dynamic case with a single cell. The reference solution is computed on a mesh of level $3 / 9$ and with $\Delta t=9.77 \cdot 10^{-9}=\Delta x_{\min } / 40$. The other solutions are calculated on a mesh of level 3/7 for decreasing $\Delta t\left(\frac{\Delta x_{\min }}{40}, \frac{\Delta x_{\min }}{80}\right.$ and $\left.\frac{\Delta x_{\min }}{160}\right)$. The system's response converges.

behavior of the system in time and selected experimentally, $\Delta t=\frac{\Delta x_{\min }}{400}$, and we impose an electric field $E=40 \mathrm{kV} / \mathrm{m}$ in the $z$-direction. The Octree has a resolution $5 / 10$, leading to a Voronoi mesh with 2,219,552 cells. The permeabilization of the cells averaged on each of the slices in the $z$-direction is presented in figure 12 . We observe a shadowing effect on the middle slice, which presents a lower degree of permeabilization. The same setup with $5 \times 5 \times 5$ cells exhibits a more pronounced shadowing, as depicted in figure in figure 13. The tree this time is level 5/9, leading to 2,614,488 Voronoi cells. Figure 14 summarizes this section by showing the average permeabilization of the entire cluster of cells for different densities of cells, from a single cell to a $5 \times 5 \times 5$ array, demonstrating the effect of shadowing. Such results are consistent with the experimental observations of Pucihar et al. [31], in which dense cell suspensions increase shadowing effects. This example demonstrates the ability to study quantitatively shadowing effects with respect to the density of cell suspensions.

\subsection{Influence of the shape}

We propose to investigate the influence of the cells' geometry on the poration. We choose an array of $N^{3}$ cells spread evenly in a box of size $1 \mathrm{~cm}^{3}$ located in a computational domain of size $2 \mathrm{~cm}^{3}$ and apply a electric potential in the $z$-direction with magnitude $40 \mathrm{kV}$. We select three shapes: spheres with radii $50 \mu \mathrm{m}$, oblate ellipsoids with radii $46 \mu \mathrm{m}$ and prolate ellipsoids with radii $53 \mu \mathrm{m}$. Here, an ellipsoid with radius $r_{0}$ is given by:

$$
\frac{x^{2}}{a^{2}}+\frac{y^{2}}{b^{2}}+\frac{z^{2}}{c^{2}}=r_{0}^{2} .
$$

It is oblate for $a=b>c$ and prolate for $a=b<c$. The coefficients $a, b$ and $c$ are chosen such that the surface of the ellipsoidal cells is the same than that of the spherical cells, and $a=b=\frac{1}{c}$ or $\frac{1}{a}=$ $\frac{1}{b}=c$, leading to $a \approx 1.225878$ for the oblate case and $a \approx 1.259835$ for the prolate case. Figure 15 

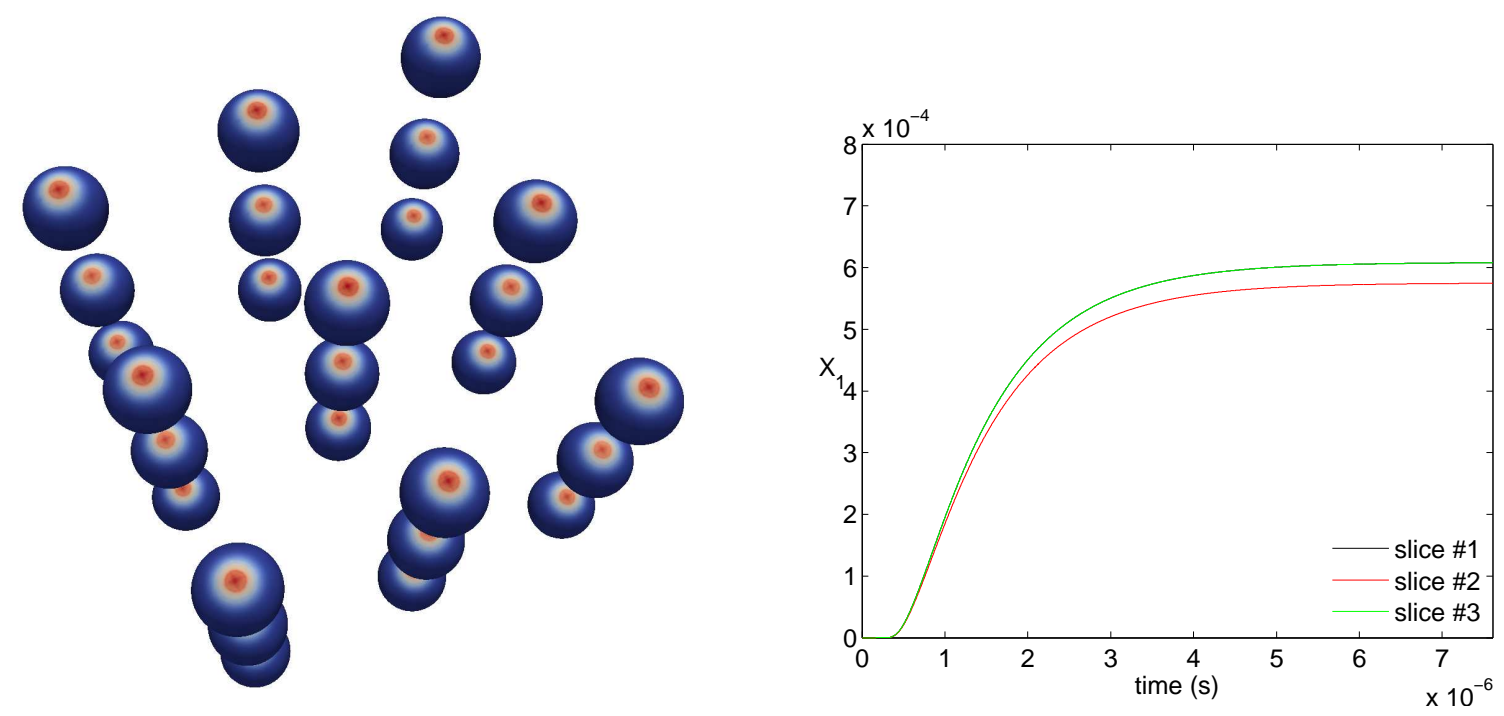

Figure 12: Left: Visualization of the permeabilization $X_{1}$ of a $3 \times 3 \times 3$ array of cells. Right: Evolution in time of the average permeabilization of the cell membranes for each $z$-slice of the $3 \times 3 \times 3$ array of cells. Note that due to symmetry the curves for the first and third slices are superposed.
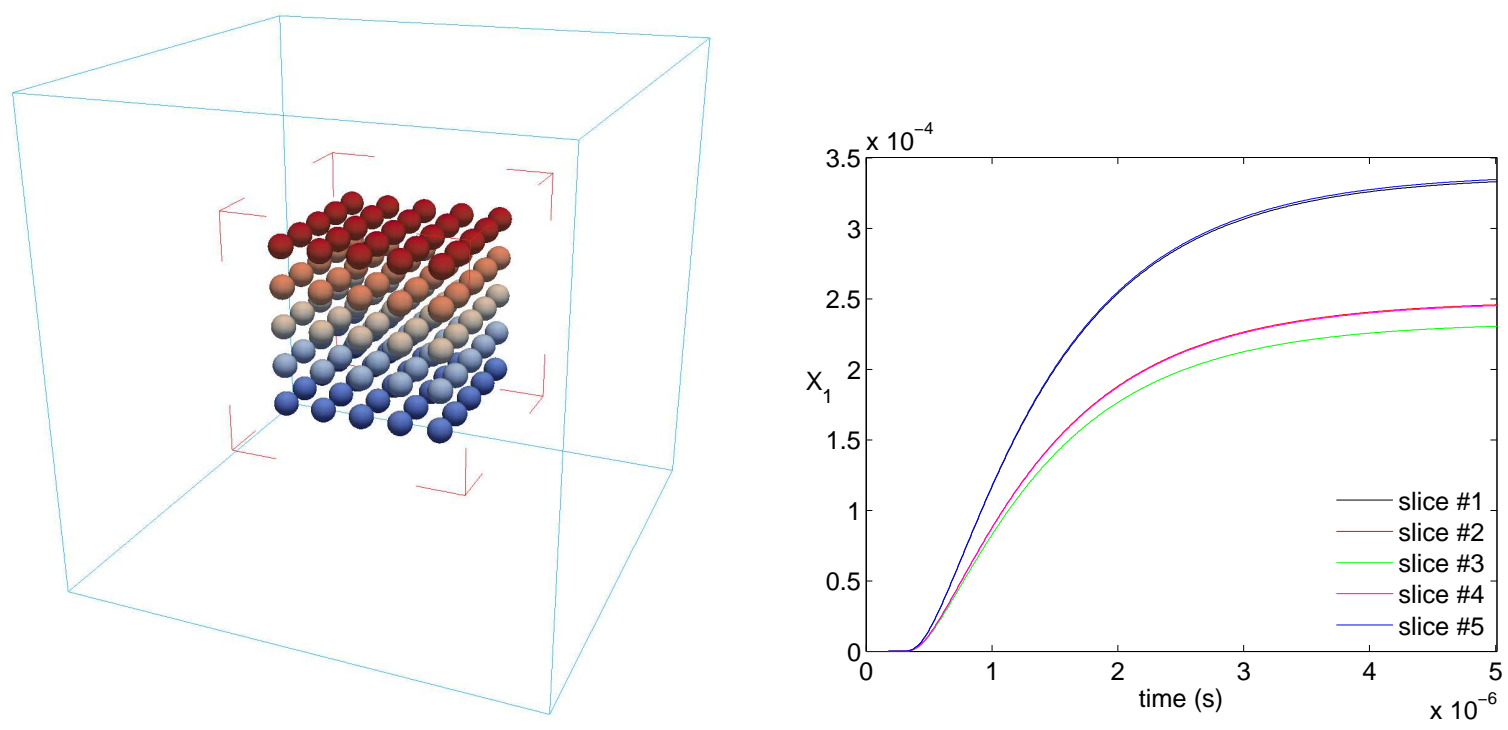

Figure 13: Left: setup for the simulation 5.1. The blue box represents the computational domain and the red corners mark the inner box in which the cells are located. The cells are colored with the electric potential $u$. Right: average permeabilization $X_{1}$ by $z$-slice as a function of time. The first and fifth slices are superposed due to symmetry, and so are the second and the fourth slices.

provides a visualization of the three shapes. The tree is level 5/9, leading to approximately 610,784 Voronoi cells for the spherical cells, 597,968 Voronoi cells for the oblate cells and 561,176 Voronoi cells for the prolate cells. The time step is set to $\Delta t=\frac{\Delta x_{\min }}{400} \approx 9.77 \cdot 10^{-9} \mathrm{~s}$. The relevant physical 


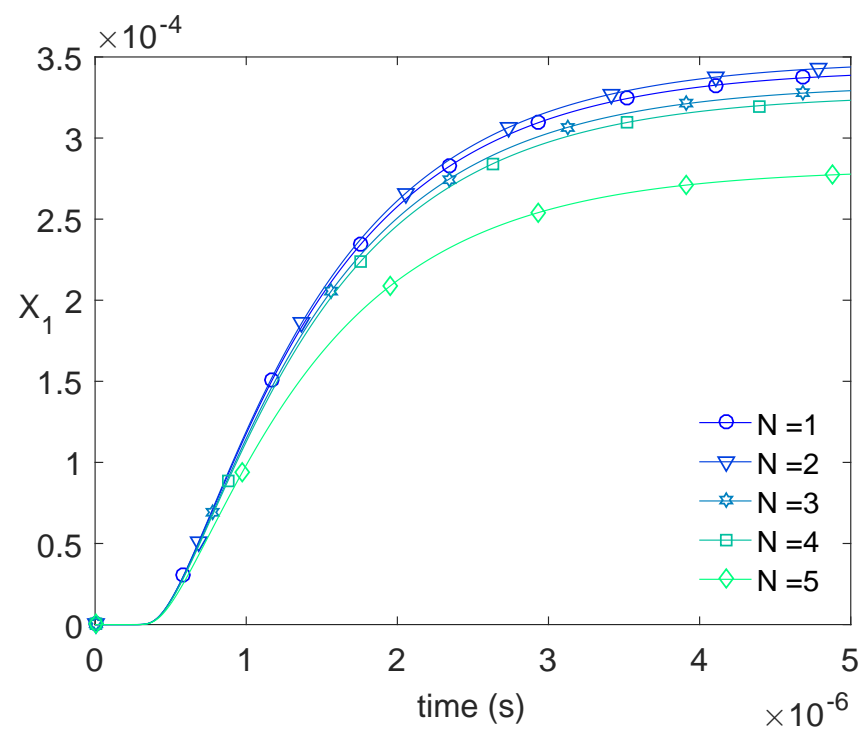

Figure 14: Influence of the cell packing on the average permeabilization $X_{1}$. A packing of $N$ corresponds to a $N \times N \times N$ array of spherical cells. As $N$ increases and the cells get closer, the shadowing effects become more pronounced and the permeabilization decreases.

parameters, i.e. the average conductance $S$, the average discontinuity in the electric potential $[u]$ at the tip of the cells, the average poration $X_{0}$ and the average permeabilization $X_{1}$, are represented in figures 16 and 17 for $N=3$ and $N=5$ respectively. We observe a strong influence of the cell shapes, with orders of magnitude of difference in the case of the permeabilization $X_{1}$. These results are consistent with the biological experiments demonstrating that, in order to increase the efficacy of electroporation in muscles, the electric field has to be applied orthogonally to the fibers [35]. This computational example demonstrates that the approach described in this manuscript is capable of studying the influence of cells' geometries. In turn, this will enable the study of electroporation in more complex cell distributions, such as in brain tissue for example, and will help determine the optimal distribution of the electric field for maximizing cell electroporation.

\subsection{Random cluster with 100 cells}

For this last example, we consider 100 cells located in a box of size $1 \mathrm{~cm}^{3}$ in a domain twice that size. We impose an electric field in the $z$-direction with intensity $40 \mathrm{kV}$ and choose $\Delta t=\frac{\Delta x_{\min }}{400} \approx$ $9.77 \cdot 10^{-9} \mathrm{~s}$. The tree resolution is $5 / 9$ and the cells are ellipsoids with random eccentricities, orientations and locations. The results are depicted in figure 18 and illustrate the capacity of our solver to handle complex layouts of numerous cells. This example is interesting for biological applications, for which cells do not have exactly the same shape and volume. Yet, it is possible to determine the distribution of ellipsoidal shapes and diameters in a sample. Hence, our numerical method makes it possible to predict quantitatively the average degree of cell permeabilization as well as the distribution of permeabilized cells in such set up. 


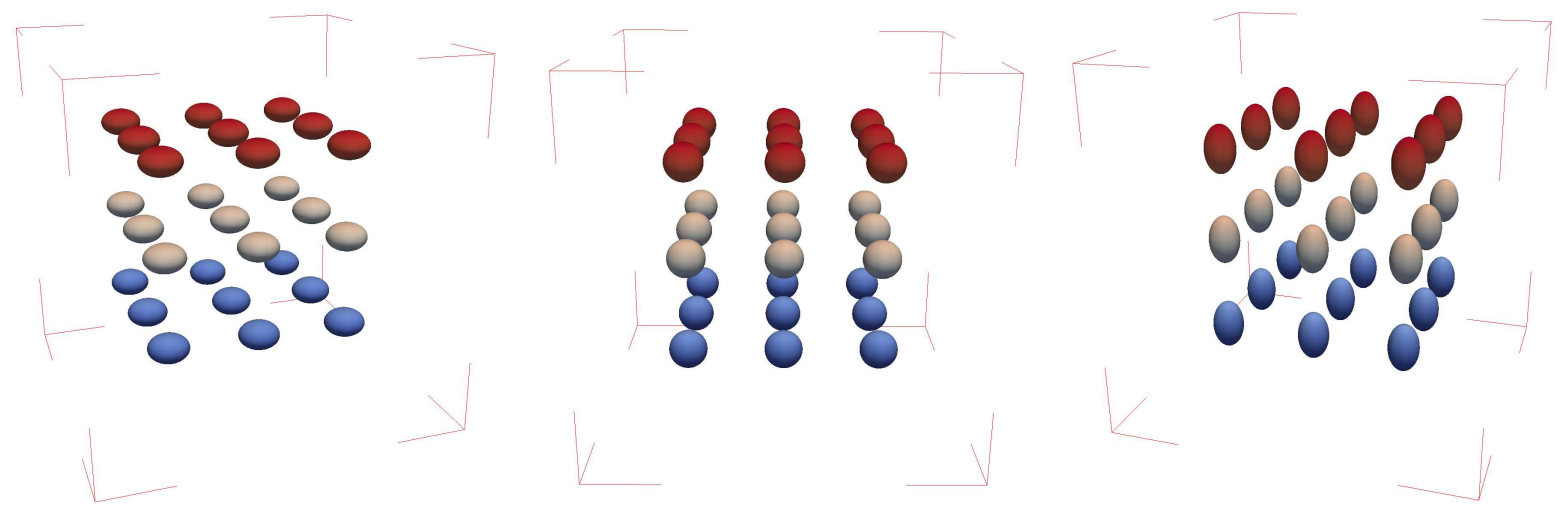

Figure 15: Visualization of an array of $3 \times 3 \times 3$ cells with oblate (left), spherical (center) and prolate (right) shapes colored with the electric potential $u$.
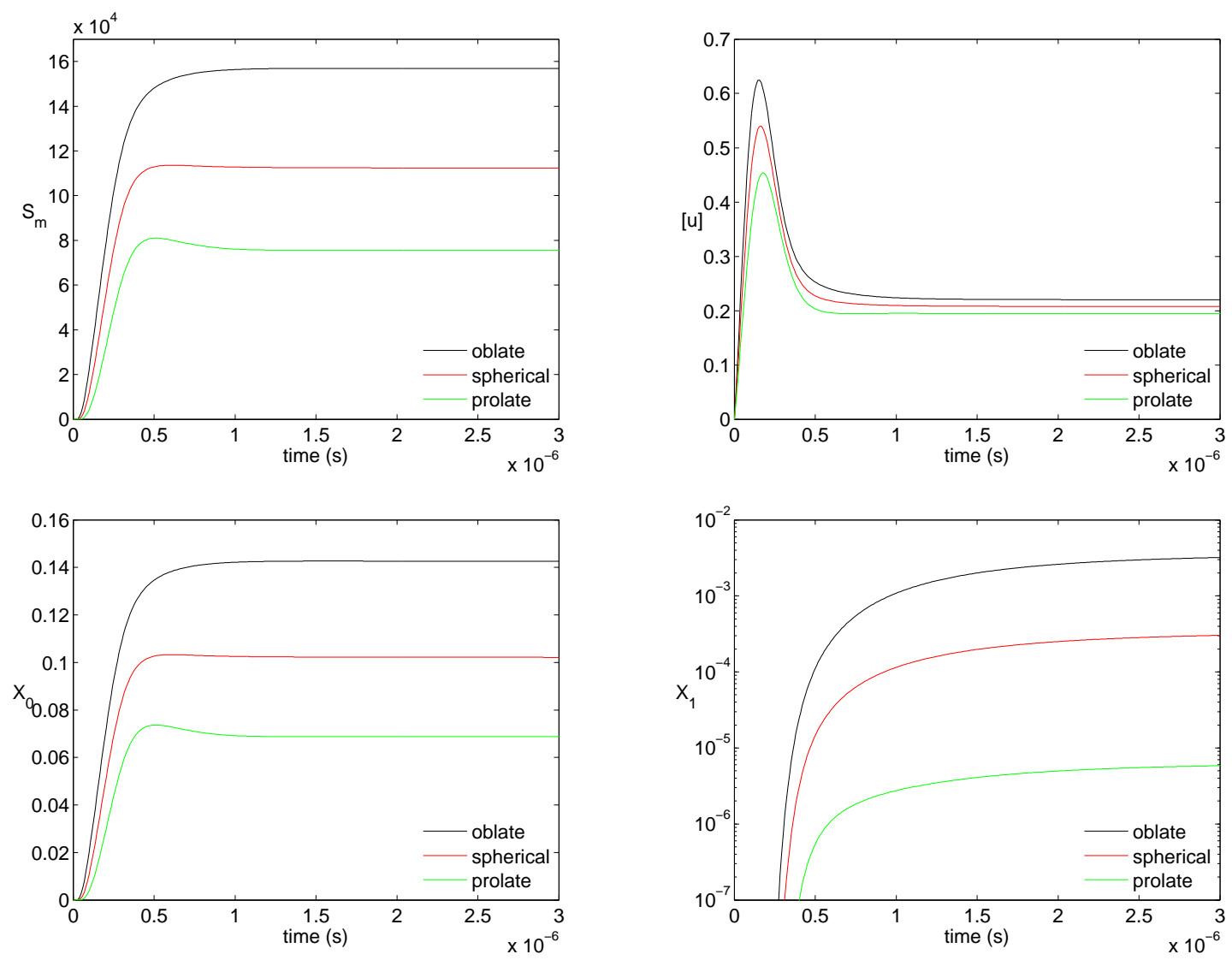

Figure 16: Representation of the relevant physical parameters as a function of time for the three different cell shapes decribed in section 5.2 and for an array of $3 \times 3 \times 3$ cells. Note that $X_{1}$ is represented with a logarithmic scale.

\section{Conclusion}

We have presented a Voronoi Interface approach for the simulation of cell electropermeabilization. In particular, we have considered a nonlinear electropermeabilization model and imposed the 

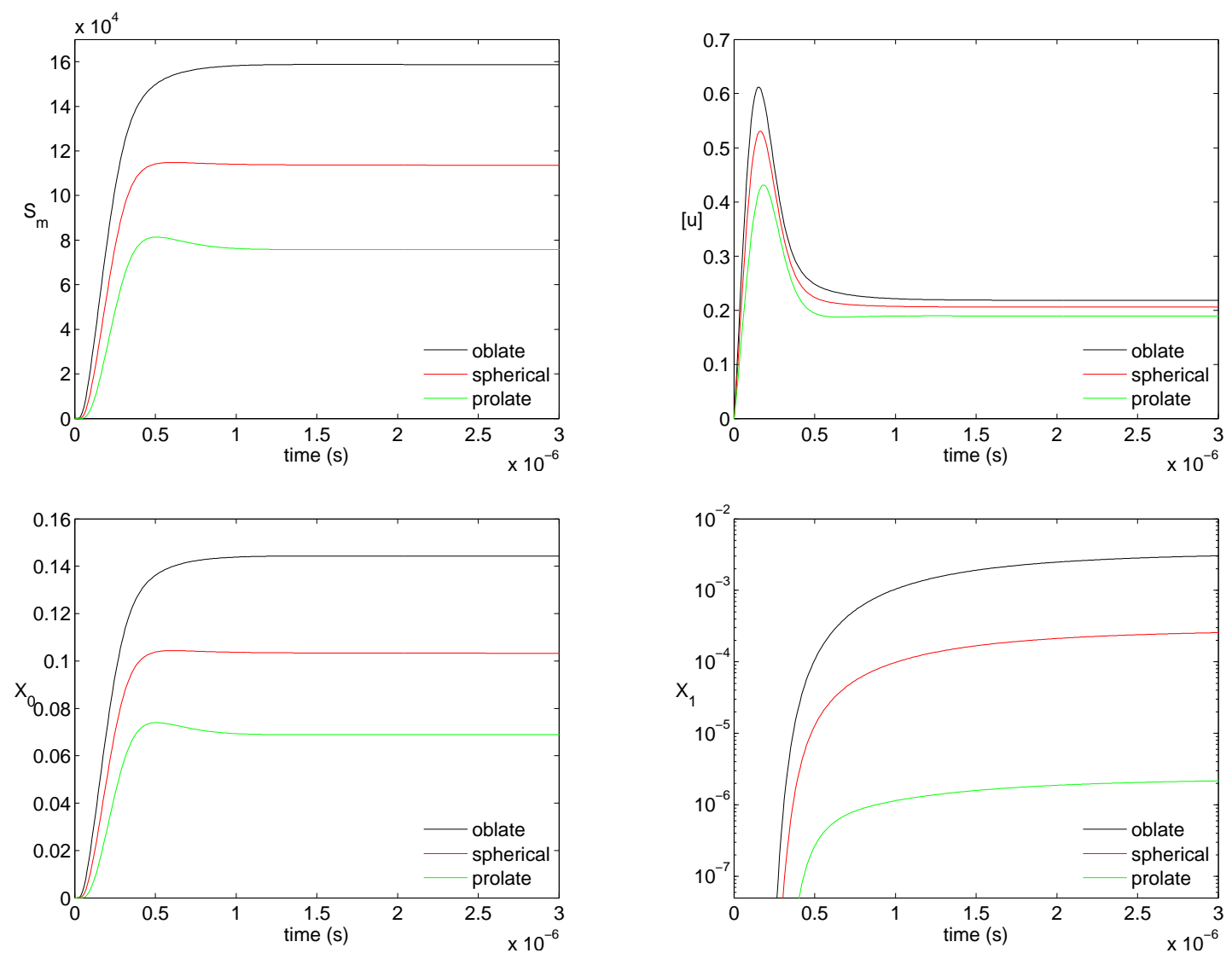

Figure 17: Representation of the relevant physical parameters as a function of time for three different cell shapes decribed in section 5.2 and for an array of $5 \times 5 \times 5$ cells. Note that $X_{1}$ is represented with a logarithmic scale.

jump condition in electrical potential in a sharp manner. The numerical treatment at the interface leads to a symmetric positive definite linear system that can be inverted efficiently. Together with the use of adaptive grids, this approach enables the study of cell aggregates. Computational experiments have illustrated the accuracy of the numerical approach and have been used to investigate the shadowing effects as well as the influence of cell's geometries on the degree of permeabilization. We find that cells with elongated shapes are more prone to be electropermeabilized if the field is orthogonal to the long axis; this is consistent with the biological experiments of Corovic et $a l$. in the context of muscles. Our work is a first-step towards studying electropermeabilization of mesoscale cell spheroids, which provide an interesting biological model of tumors. Future work will consider the extension to massively parallel architectures, which will provide a computational tool that makes it possible to compare with macroscale models obtained by either phenomenological considerations or by rigorous homogenization of a microscale single-cell model. Our approach can also serve as an advanced numerical tool that can enable the comparison between theoretical models and biological experiments of electropermeabilization of mesoscale spheroids. 

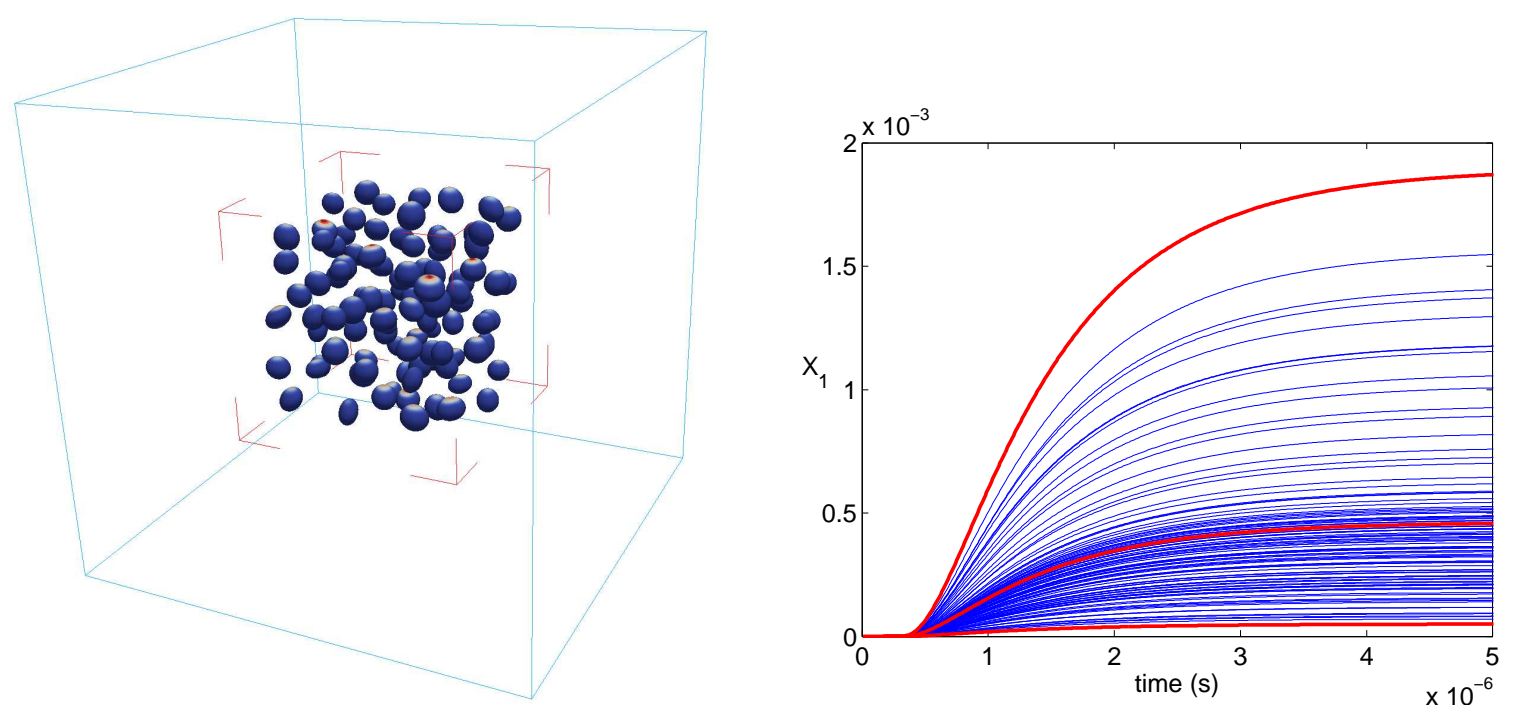

Figure 18: Representation of a cluster of 100 cells (left) and the corresponding permeabilization $X_{1}$ (right). The average, maximum and minimum levels of permeabilization are represented by the red lines.

\section{Acknowledgements}

The research of A. Guittet and F. Gibou was supported in part by ARO W911NF-16-1-0136, NSF under the DMREF program DMR-1534264, NSF DMS 1620471 and ONR N00014-11-10027. The research of C. Poignard has been carried out with financial support from the French State, managed by the French National Research Agency (ANR) in the frame of the "Investments for the future" Programme IdEx Bordeaux - CPU (ANR-10-IDEX-03-02). C.P's research is also supported by Plan Cancer DYNAMO (ref. 9749) and Plan Cancer NUMEP (ref. 11099). 


\section{References}

[1] I. Babuška. The finite element method for elliptic equations with discontinuous coefficients. Computing, 5:207-213, 1970.

[2] J. Bramble and J. King. A finite element method for interface problems in domains with smooth boundaries and interfaces. Adv. Comput. Math., 6:109-138, 1996.

[3] Marco Cisternino and Lisl Weynans. A parallel second order cartesian method for elliptic interface problems. Communications in Computational Physics, 12:1562-1587, 112012.

[4] K. DeBruin and W. Krassowska. Modelling electroporation in a single cell. I. Effects of field strength and rest potential. Biophysical Journal, 77:1213-1224, Sept 1999.

[5] M. Dryja. A neumann-neumann algorithm for mortar discretization of elliptic problems with discontinuous coefficients. Num. Math., 99:645-656, 2005.

[6] Richard E. Ewing, Zhilin Li, Tao Lin, and Yanping Lin. The immersed finite volume element methods for the elliptic interface problems. Mathematics and Computers in Simulation, 50(14):63 - 76, 1999.

[7] E.C. Fear and M.A. Stuchly. Modelling assemblies of biological cells exposed to electric fields. IEEE Trans Biomed Eng, 45(10):1259-1271, Oct 1998.

[8] R Fedkiw, T Aslam, B Merriman, and S Osher. A non-oscillatory eulerian approach to interfaces in multimaterial flows (the ghost fluid method). J. Comput. Phys., 152:457-492, 1999.

[9] R.A. Finkel and J.L. Bentley. Quad trees a data structure for retrieval on composite keys. Acta Informatica, 4(1):1-9, 1974.

[10] K.R. Foster and H.P. Schwan. Dielectric properties of tissues and biological materials: a critical review. CRC in Biomedical Engineering, 17(1):25-104, 1989.

[11] B. Gabriel and J. Teissié. Time courses of mammalian cell electropermeabilization observed by millisecond imaging of membrane property changes during the pulse. Biophys. $J ., 76(4): 2158-2165$ (electronic), 1999.

[12] Laure Gibot, Luc Wasungu, Justin Teissié, and Marie-Pierre Rols. Antitumor drug delivery in multicellular spheroids by electropermeabilization. Journal of Controlled Release: Official Journal of the Controlled Release Society, 167(2):138-147, April 2013.

[13] Yan Gong, Bo Li, and Zhilin Li. Immersed-interface finite-element methods for elliptic interface problems with nonhomogeneous jump conditions. SIAM Journal on Numerical Analysis, 46(1):472-495, 2008. 
[14] Arthur Guittet, Mathieu Lepilliez, Sebastien Tanguy, and Frederic Gibou. Solving elliptic problems with discontinuities on irregular domains - the voronoi interface method. Journal of Computational Physics, 298:747 - 765, 2015.

[15] G. Guyomarc'h, C.-O. Lee, and K. Jeon. A discontinuous Galerkin method for elliptic interface problems with application to electroporation. Comm. Numer. Methods Engrg., 25(10):991-1008, 2009.

[16] Franziska Hirschhaeuser, Heike Menne, Claudia Dittfeld, Jonathan West, Wolfgang MuellerKlieser, and Leoni A. Kunz-Schughart. Multicellular tumor spheroids: an underestimated tool is catching up again. Journal of Biotechnology, 148(1):3-15, July 2010.

[17] J. Huang and J. Zou. A mortar element method for elliptic problems with discontinuous coefficients. IMA J. Numer. Anal., 22:549-576, 2001.

[18] O. Kavian, M. Leguèbe, C. Poignard, and L. Weynans. "Classical" electropermeabilization modeling at the cell scale. J. Math. Biol., 68(1-2):235-265, 2014.

[19] M. Leguèbe, C. Poignard, and L. Weynans. A second-order Cartesian method for the simulation of electropermeabilization cell models. J. Comput. Phys., 292:114-140, 2015.

[20] M. Leguèbe, A. Silve, L.M. Mir, and C. Poignard. Conducting and permeable states of cell membrane submitted to high voltage pulses: Mathematical and numerical studies validated by the experiments. Journal of Theoretical Biology, 360:83-94, 2014. cited By 0.

[21] R LeVeque and Z Li. The immersed interface method for elliptic equations with discontinuous coefficients and singular sources. SIAM Journal of Numerical Analysis, 31:1019-1044, 1994.

[22] Zhilin Li, Tao Lin, and Xiaohui Wu. New cartesian grid methods for interface problems using the finite element formulation. Numerische Mathematik, 96(1):61-98, 2003.

[23] X D Liu, R Fedkiw, and M Kang. A boundary condition capturing method for poisson's equation on irregular domains. J. Comput. Phys., 154:151, 2000.

[24] Xu-Dong Liu and Thomas Sideris. Convergence of the ghost-fluid method for elliptic equations with interfaces. Math. Comp., 72:1731-1746, 2003.

[25] Donald Meagher. Geometric modeling using octree encoding. Computer Graphics and Image Processing, 19(2):129 - 147, 1982.

[26] C. Min. Local level set method in high dimension and codimension. J. Comput. Phys., 200:368-382, 2004.

[27] L.M. Mir. Therapeutic perspectives of in vivo cell electropermeabilization. Bioelectrochemistry, 53:1-10, 2001. 
[28] Mohammad Mirzadeh, Maxime Theillard, and Frederic Gibou. A Second-Order Discretization of the Nonlinear Poisson-Boltzmann Equation over Irregular Geometries using NonGraded Adaptive Cartesian Grids. Journal of Computational Physics, 230(5):2125-2140, December 2010.

[29] S. Osher and J.A. Sethian. Fronts propagating with curvature-dependent speed: Algorithms based on hamilton-jacobi formulations. Journal of Computational Physics, 79:12-49, 1988.

[30] C. Poignard, A. Silve, and L. Wegner. Different approaches used in modeling of cell membrane electroporation. In D. Miklavčič, editor, Handbook on Electroporation. Springer, To appear.

[31] Gorazd Pucihar, Tadej Kotnik, Justin Teissié, and Damijan Miklavčič. Electropermeabilization of dense cell suspensions. European Biophysics Journal, 36(3):173-185, 2007.

[32] J. Strain. Tree methods for moving interfaces. J. Comput. Phys., 151:616-648, 1999.

[33] J. Teissié. Electropermeabilization of the cell membrane. In S. Li, J. Cutrera, R. Heller, and J. Teissié, editors, Electroporation Protocols. Preclinical and Clinical Gene Medicine, chapter 2. Springer, 2014.

[34] J. Teissié, M. Golzio, and M.P. Rols. Mechanisms of cell membrane electropermeabilization: A minireview of our present (lack of ?) knownledge. Biochimica et Biophysica Acta, 1724:270-280, 2005.

[35] Selma Črović, Anže Z̆upanič, Simona Kranjc, Bassim Al Sakere, Anne Leroy-Willig, Lluis M. Mir, and Damijan Miklavčič. The influence of skeletal muscle anisotropy on electroporation: in vivo study and numerical modeling. Medical \& Biological Engineering \& Computing, 48(7):637-648, July 2010.

[36] Z. Vasilkoski, A. T. Esser, T. R. Gowrishankar, and J. C. Weaver. Membrane electroporation: The absolute rate equation and nanosecond time scale pore creation. Phys Rev E Stat Nonlin Soft Matter Phys, 74(2 Pt 1):021904, Aug 2006.

[37] M.C. Vernhes, P.A. Cabanes, and J. Teissié. Chinese hamster ovary cells sensitivity to localized electrical stresses. Bioelectrochem. Bioenerg., 48:17-25, 1999.

[38] J.C Weaver. Electroporation of cells and tissues. IEEE Trans. on Plasma Sci., 28, 2000. 